\title{
High yielding synthesis of $N$-ethyl dehydroamino acids
}

\author{
Luís S. Monteiro* and Ana S. Suárez \\ Chemistry Centre, University of Minho, Gualtar, 4710-057 Braga, Portugal \\ Author's address: L. S. Monteiro, Chemistry Centre, University of Minho, Gualtar, 4710-057 Braga, Portugal, \\ Fax: +351 253604382, \\ e-mail: monteiro@quimica.uminho.pt.
}

\begin{abstract}
Recently we reported the use of a sequence of alkylation and dehydration methodologies to obtain $N$-ethyl- $\alpha, \beta$ dehydroamino acid derivatives. The application of this $N$-alkylation procedure to several methyl esters of $\beta$, $\beta$-dibromo and $\beta$ bromo, $\beta$-substituted dehydroamino acids protected with standard amine protecting groups was subsequently reported. The corresponding $N$-ethyl, $\beta$-bromo dehydroamino acid derivatives were obtained in fair to high yields and some were used as substrates in Suzuki cross coupling reactions to give $N$-ethyl, $\beta, \beta$-disubstituted dehydroalanine derivatives.

Herein, we further explore $N$-ethylation of $\beta$-halo dehydroamino acid derivatives using triethyloxonium tetrafluoroborate as alkylating agent but substituting $N, N$-diisopropylethylamine for potassium tert-butoxide as auxiliary base. In these conditions, for all $\beta$-halo dehydroamino acid derivatives, reactions were complete and the $N$-ethylated derivative could be isolated in high yield. This method was also applied for $N$-ethylation of non-halogenated dehydroamino acids. Again, with all compounds the reactions were complete and the $N$-ethyl dehydroamino acid derivatives could be isolated in high yields. Some of these $N$-ethyl dehydroamino acid methyl ester derivatives were converted in high yields to their corresponding acids and coupled to an amino acid methyl ester to give $N$-ethyl dehydrodipeptide derivatives in good yields.

Thus, this method constitutes a general procedure for high yielding synthesis of $N$-ethylated dehydroamino acids, which can be further applied in peptide synthesis.
\end{abstract}

Keywords: Amino acids - Nonnatural amino acids - Alkylation - $N$-Ethyl dehydroamino acids - Protecting groups. 


\section{Introduction}

Non-proteinogenic amino acids are an important class of organic compounds with a large application spectrum in medicinal chemistry, since they can have intrinsic biological activity or can be found in peptides with antiviral, antitumor, anti-inflammatory or immunosuppressive activities. Among non-proteinogenic amino acids are $\mathrm{N}$-alkylamino acids and dehydroamino acids, both of which can be found in many biologically important peptides (Gilon et al. 2003; Kotha 2003; Rilatt et al. 2005). N-Alkylamino acids are constituents of various important naturally occurring peptides and proteins (Gilon et al. 2003; Kotha 2003; Rilatt et al. 2005). Several $N$-alkylated peptides show antibiotic, anticancer or antiviral activity (Wenger 1985). $N$-Alkylation of the peptide bond causes changes in volume and conformation of peptides, resulting in reduced flexibility, increase of permeability for the membrane (increased lipophilicity) and prevention of cleavage by proteolytic enzymes (Aurelio et al. 2004). Dehydroamino acids are outstanding compounds as they are key intermediates in amino acid and peptide synthesis, having been found in naturally occurring peptides of fungal and microbial origin (Aydin et al. 1985) and from marine organisms (Hamann and Scheuer 1993; Goetz et al. 1997), in which they contribute with a catalytic role in the active sites of some enzymes. They are also found in a variety of peptide antibiotics of bacterial origin that include the lantibiotics (nisin, epidermin, subtilin, gallidermin) (Palmer et al. 1992; Jung 1991; Chatterjee et al. 2005).

Many methods of synthesis of $N$-alkylamino acids have been developed, most of them are $N$-methylation (Aurelio et al. 2004). Recently, Liguori et al. (2010) proposed the ethylation of several 4-nitrobenzenesulfonyl (Nosyl) protected amino acids using triethyloxonium tetrafluoroborate $\left(\mathrm{Et}_{3} \mathrm{OBF}_{4}\right)$ as alkylating agent and $\mathrm{N}, \mathrm{N}$ diisopropylethylamine (DIPEA) as base to give $N$ ethylamino acid derivatives in high yields. Subsequently, we reported the use of a combination of this alkylation procedure and dehydration methodologies previously developed by us (Ferreira et al. 1999; Ferreira et al. 2007) to obtain new non-proteinogenic amino acids namely, $\mathrm{N}$-(4nitrophenylsulfonyl), $\quad N$-ethyl- $\alpha, \beta$-dehydroamino acids (Monteiro et al. 2010). Thus, it was possible to obtain for the first time, new non-natural amino acids which incorporate both the $N$-ethyl and the $\alpha, \beta$-dehydro moieties.

In the case of $N$-alkylation of dehydroamino acids, an alternative to the electron withdrawing effect of the amine protecting group could be the presence of electron withdrawing substituents on the $\beta$-carbon atom. Thus, we tested the triethyloxonium tetrafluoroborate / N,Ndiisopropylethylamine $N$-alkylation procedure with several methyl esters of $\beta, \beta$-dibromo and $\beta$-bromo, $\beta$-substituted dehydroamino acids protected with standard amine protecting groups such as tert-butyloxycarbonyl (Boc), benzyloxycarbonyl (Z) and 4-nitrobenzyloxycarbonyl $\left[\mathrm{Z}\left(\mathrm{NO}_{2}\right)\right]$ as well as acyl and sulfonyl amine protecting groups (Monteiro et al. 2011). Depending on the nature of the halogenated dehydroamino acid and of the protecting group, variable ratios of product to reactant were obtained. These ranged from total conversion to product $[\beta, \beta$ dibromo, dehydroalanine derivatives protected with $\mathrm{Z}\left(\mathrm{NO}_{2}\right)$ and $\mathrm{Z}, \quad \beta$-bromo dehydroaminobutyric acid and dehydrophenylalanine derivatives protected with Nosyl and Tos] to complete absence of reaction [the methyl ester of $N$ (tert-butyloxycarbonyl), $\beta$-bromo dehydroaminobutyric acid]. Some of these $N$-ethylated dehydroamino acid derivatives were used as substrates in cross coupling reactions to give the methyl esters of $N$-protected, $N$-ethyl, $\beta, \beta$-disubstituted dehydroalanines.

Herein, we further explore $N$-ethylation of $\beta$-halo dehydroamino acid derivatives substituting DIPEA for potassium tert-butoxide as auxiliary base. Contrary to reactions carried out using DIPEA in which no reaction or incomplete reactions were observed, with the use of tertbutoxide, all reactions were complete giving $N$-ethyl dehydroamino acid derivatives in high yields. The method is further applied to non-halogenated dehydroamino acid derivatives.

\section{Materials and methods}

Melting points were determined with a Gallenkamp apparatus and are uncorrected. ${ }^{1} \mathrm{H}$ and ${ }^{13} \mathrm{C}$ NMR spectra were recorded with a Varian Unity Plus spectrometer at 300 and $75.4 \mathrm{MHz}$, respectively, or with a Bruker Avance $\mathrm{II}^{+}$ operating at 400 and $100.6 \mathrm{MHz}$, respectively. ${ }^{1} \mathrm{H}-{ }^{1} \mathrm{H}$ spinspin decoupling, DEPT $\left(\theta 45^{\circ}\right)$, HMQC and HMBC were used to attribute some signals. The stereochemistry of the dehydroamino acids derivatives was determined by NOE difference experiments. Chemical shifts are given in ppm and coupling constants $(J)$ in Hz. HRMS data were obtained by the mass spectrometry service of the University of Vigo, Spain. Elemental analysis was performed with a LECO CHNS 932 elemental analyzer. The reactions were monitored by thin layer chromatography (TLC). Petroleum ether refers to fractions with the boiling range $40-60{ }^{\circ} \mathrm{C}$. Solvents were used without purification except for acetonitrile and dichloromethane, which were dried according to standard procedures.

Synthesis of the methyl esters of $N$-protected $\beta$ hydroxyamino acids

Synthesis of compounds $\mathrm{Z}\left(\mathrm{NO}_{2}\right)$-L-Ser-OMe (Ferreira et al. 1999), Z-L-Ser-OMe (Ferreira et al. 1999), Boc-L-Ser-OMe (Ferreira et al. 1999), Z(NO 2$)$-L-Thr-OMe (Ferreira et al. 1999), Z-L-Thr-OMe (Ferreira et al. 1999), Boc-L-Thr-OMe (Ferreira et al. 1999), Tos-L-Thr-OMe (Ferreira et al. 1999), $\mathrm{Z}\left(\mathrm{NO}_{2}\right)$-D,L-Phe $(\beta-\mathrm{OH})-\mathrm{OMe}$ (Ferreira et al. 1999), Z-D,L$\mathrm{Phe}(\beta-\mathrm{OH})-\mathrm{OMe}$ (Monteiro et al. 2011), Boc-D,L-Phe $(\beta$ $\mathrm{OH})-\mathrm{OMe}$ (Ferreira et al. 2001), Tos-D,L-Phe( $\beta-\mathrm{OH})-\mathrm{OMe}$ (Ferreira et al. 2007)

The synthesis of these compounds was described in the references given above.

Synthesis of the methyl esters of $N$-protected dehydroamino acids

Synthesis of compounds $\mathrm{Z}\left(\mathrm{NO}_{2}\right)-\Delta$ Ala-OMe (1a) (Ferreira et al. 2007), Z- $\Delta$ Ala-OMe (1b) (Bertj et al. 1992), Boc$\triangle$ Ala-OMe (1c) (Ferreira et al. 1999), Z(NO$\left.{ }_{2}\right)-\mathrm{Z}-\Delta \mathrm{Abu}-$ OMe (Z-2a) (Ferreira et al. 1999), Z-Z- $\Delta$ Abu-OMe (Z-2b) (Ferreira et al. 1999), Boc-Z- $\Delta$ Abu-OMe (Z-2c) (Ferreira et al. 1999), Tos-Z- $\Delta$ Abu-OMe (Z-2d) (Ferreira et al. 2007), $\mathrm{Z}\left(\mathrm{NO}_{2}\right)$-Z- $\Delta$ Phe-OMe (Z-3a) (Monteiro et al. 2011), Z-Z$\Delta$ Phe-OMe (Z-3b) (Monteiro et al. 2011), Boc-Z- $\Delta$ Phe-OMe (Z-3c) (Ferreira et al. 2001), Tos-Z- $\Delta$ Phe-OMe (Z-3d) (Ferreira et al. 2007).

The synthesis of these compounds was described in the references given above. 
Synthesis of the methyl esters of $N$-protected $\beta$-halo dehydroamino acids

Synthesis of compounds Boc- $\Delta \mathrm{Ala}(\beta, \beta-\mathrm{Br})-\mathrm{OMe}(\mathbf{4 c})$ (Silva et al. 2002), $\mathrm{Z}\left(\mathrm{NO}_{2}\right)-E-\Delta \mathrm{Abu}(\beta-\mathrm{Br})-\mathrm{OMe}(\mathrm{E}-5 \mathbf{a})$ (Ferreira et al. 2007), $\mathrm{Z}\left(\mathrm{NO}_{2}\right)-\mathrm{Z}-\Delta \mathrm{Abu}(\beta-\mathrm{Br})-\mathrm{OMe}(\mathrm{Z}-5 \mathbf{a})$ (Ferreira et al. 2007), Z-Z- $\triangle \mathrm{Abu}-(\beta-\mathrm{Br}) \mathrm{OMe}(\mathrm{Z}-5 \mathbf{b})$ (Ferreira et al. 2007), Boc-E- $\Delta \mathrm{Abu}(\beta-\mathrm{Br})-\mathrm{OMe}(\mathrm{E}-5 \mathrm{c})$ (Silva et al. 2002), Boc-Z- $\Delta \mathrm{Abu}(\beta-\mathrm{Br})-\mathrm{OMe}(\mathrm{Z}-5 \mathrm{c})$ (Silva et al. 2002), $\mathrm{Z}\left(\mathrm{NO}_{2}\right)-$ $Z-\Delta \mathrm{Phe}(\beta-\mathrm{Br})-\mathrm{OMe}$ (Z-6a) (Monteiro et al. 2011), Z-Z$\Delta \mathrm{Phe}(\beta-\mathrm{Br})-\mathrm{OMe}$ (Z-6b) (Monteiro et al. 2011), Boc-Z$\Delta \mathrm{Phe}(\beta-\mathrm{Br})-\mathrm{OMe}$ (Z-6c) (Abreu et al. 2004), Boc-Z- $\Delta \mathrm{Phe}(\beta$ I)-OMe (Z-7c) (Queiroz et al. 2008).

The synthesis of these compounds was described in the references given above.

Synthesis of $\mathrm{Z}\left(\mathrm{NO}_{2}\right)-\mathrm{Z}-\Delta P h e(\beta-\mathrm{I})-\mathrm{OMe}(\mathrm{Z}-\mathrm{7a}) \quad \mathrm{Z}\left(\mathrm{NO}_{2}\right)-\mathrm{Z}$ $\triangle$ Phe-OMe (Z-3a) (1.069 g, $3.000 \mathrm{mmol})$ was dissolved in

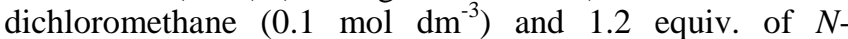
iodosuccinimide was added with vigorous stirring. After reacting for 16 hours, triethylamine (1.5 equiv.) was added and stirring continued for an additional hour. An additional $100 \mathrm{~cm}^{3}$ of dichloromethane were added and the organic phase was washed with $\mathrm{KHSO}_{4}\left(1 \mathrm{~mol} \mathrm{dm}^{-3}\right), \mathrm{NaHCO}_{3}$ $\left(1 \mathrm{~mol} \mathrm{dm}^{-3}\right)$ and brine (3 times $30 \mathrm{~cm}^{3}$ each). After drying over $\mathrm{MgSO}_{4}$ the extract was taken to dryness at reduced pressure to afford Z-7a $(1.230 \mathrm{~g}, 85 \%)$ as a light yellow solid. M.p. $135.0-136.0^{\circ} \mathrm{C}$ (from ethyl acetate $/ n$-hexane). ${ }^{1} \mathrm{H}$ NMR (300 MHz, $\mathrm{CDCl}_{3}$ ): $\delta=3.46$ (br. s, $3 \mathrm{H}, \mathrm{CH}_{3} \mathrm{OMe}$ ), 5.28 [s, $2 \mathrm{H}, \mathrm{CH}_{2} \mathrm{Z}\left(\mathrm{NO}_{2}\right)$ ], 6.69 (br. $\mathrm{S}, 1 \mathrm{H} \mathrm{NH}$ ), 7.27-7.35 $(\mathrm{m}, 5 \mathrm{H}, \operatorname{ArH}), 7.54(\mathrm{~d}, J=8.4 \mathrm{~Hz}, 2 \mathrm{H}, \operatorname{ArH}), 8.24(\mathrm{~d}, J=$ $8.4 \mathrm{~Hz}, 2 \mathrm{H}, \mathrm{ArH}) \mathrm{ppm} .{ }^{13} \mathrm{C} \mathrm{NMR}\left(75.4 \mathrm{MHz}, \mathrm{CDCl}_{3}\right): \delta=$ $52.53\left(\mathrm{OCH}_{3}\right), 66.33\left[\mathrm{CH}_{2} \mathrm{Z}\left(\mathrm{NO}_{2}\right)\right], 95.39(\mathrm{C}), 123.79(\mathrm{CH})$, $128.23(\mathrm{CH}), 128.37(\mathrm{CH}), 128.61(\mathrm{CH}), 129.01(\mathrm{CH})$, 132.95 (C), 140.34 (C), 142.53 (C), 147.77 (C), 152.69 $(\mathrm{C}=\mathrm{O}), 163.93(\mathrm{C}=\mathrm{O})$ ppm. $\mathrm{C}_{18} \mathrm{H}_{15} \mathrm{~N}_{2} \mathrm{O}_{6} \mathrm{I}(482.23)$ : calcd. $\mathrm{C}$ 44.83, H 3.14, N 5.81; found C 44.70, H 3.15, N 6.22.

Synthesis of the methyl esters of $N$-(tert-butyloxycarbonyl), $N$-ethyl- $\beta, \beta$-dibromodehydroalanine and $N$-protected, $N$ ethyl, $\beta$-substituted, $\beta$-halodehydroamino acids

General procedure for the synthesis of the methyl esters of $N$-(tert-butyloxycarbonyl), $N$-ethyl- $\beta, \beta$ dibromodehydroalanine and N-protected, N-ethyl, $\beta$ substituted, $\beta$-halodehydroamino acids

The methyl ester of the $N$-protected, $\beta$-halodehydroamino acid was dissolved in anhydrous dichloromethane $(0.05 \mathrm{~mol}$ $\mathrm{dm}^{-3}$ ) followed by addition of 3.5 equiv. of potassium tertbutoxide and 2.5 equiv. of triethyloxonium tetrafluoroborate, under inert atmosphere. The reaction mixture was stirred at room temperature for 30 minutes. Then dichloromethane was added $\left(50 \mathrm{~cm}^{3}\right)$ and the organic phase was washed with $\mathrm{KHSO}_{4}\left(1 \mathrm{~mol} \mathrm{dm}^{-3}\right), \mathrm{NaHCO}_{3}$ $\left(1 \mathrm{~mol} \mathrm{dm}^{-3}\right)$ and brine (3 times $20 \mathrm{~cm}^{3}$ each), dried over $\mathrm{MgSO}_{4}$ and the solvent evaporated at reduced pressure.

Synthesis of Boc-N(Et)- $\triangle A l a(\beta, \beta-B r)-O M e(8 c)$ The general procedure described above was followed using Boc$\Delta \mathrm{Ala}(\beta, \beta-\mathrm{Br})-\mathrm{OMe}(\mathbf{4 c})(0.066 \mathrm{~g}, 0.174 \mathrm{mmol})$ as reactant to afford 8c (Monteiro et al. 2011) (0.066 g, 93\%).

Synthesis of $Z\left(N_{2}\right)-N(E t)-E-\triangle A b u(\beta-B r)-O M,(E-9 a)$ The general procedure described above was followed using $\mathrm{Z}\left(\mathrm{NO}_{2}\right)-E-\Delta \mathrm{Abu}(\beta-\mathrm{Br})-\mathrm{OMe}(E-5 \mathbf{a})(0.065 \mathrm{~g}, 0.175 \mathrm{mmol})$ as reactant to afford $E$-9a (Monteiro et al. 2011) (0.066 g, $94 \%)$.
Synthesis of $Z\left(N_{2}\right)-N(E t)-Z-\triangle A b u(\beta-B r)-O M e$ (Z-9a) The general procedure described above was followed using $\mathrm{Z}\left(\mathrm{NO}_{2}\right)-Z-\Delta \mathrm{Abu}(\beta-\mathrm{Br})-\mathrm{OMe}(Z-5 \mathbf{a})(0.039 \mathrm{~g}, 0.105 \mathrm{mmol})$ as reactant to afford Z-9a (Monteiro et al. 2011) (0.035 g, $82 \%)$.

Synthesis of Z-N(Et)-Z- $\triangle A b u(\beta-B r)-O M e(Z-9 b)$ The general procedure described above was followed using $Z-Z$ $\triangle \mathrm{Abu}(\beta-\mathrm{Br})-\mathrm{OMe}(Z-5 \mathbf{b})(0.033 \mathrm{~g}, 0.100 \mathrm{mmol})$ as reactant to afford Z-9b (Monteiro et al. 2011) $(0.031 \mathrm{~g}, 86 \%)$.

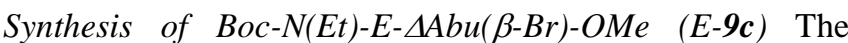
general procedure described above was followed using Boc$E-\Delta \mathrm{Abu}(\beta-\mathrm{Br})-\mathrm{OMe} \quad(E-5 \mathrm{c}) \quad(0.221 \mathrm{~g}, \quad 0.750 \mathrm{mmol})$ as reactant to afford $E-9 \mathrm{c}(0.215 \mathrm{~g}, 89 \%)$ as a colorless oil that failed to crystallize. ${ }^{1} \mathrm{H} \mathrm{NMR}\left(400 \mathrm{MHz}, \mathrm{CDCl}_{3}\right)$ : (rotamers) $\delta=1.00-1.21\left(\mathrm{~m}, 3 \mathrm{H}, \mathrm{CH}_{2} \mathrm{CH}_{3}\right), 1.39-1.48\left(\mathrm{~m}, 9 \mathrm{H}, \mathrm{CH}_{3}\right.$ Boc), 2.40 (s, $\left.3 \mathrm{H}, \gamma \mathrm{CH}_{3}\right), 3.40$ (br. q, $J=7.2 \mathrm{~Hz}, 2 \mathrm{H}$, $\left.\mathrm{CH}_{2} \mathrm{CH}_{3}\right), 3.75,3.77\left(2 \mathrm{~s}, 3 \mathrm{H}, \mathrm{CH}_{3} \mathrm{OMe}\right) \mathrm{ppm} .{ }^{13} \mathrm{C} \mathrm{NMR}$ $\left(100.6 \mathrm{MHz}, \mathrm{CDCl}_{3}\right)$ : (rotamers) $\delta=12.98,13.47\left(\mathrm{CH}_{3}\right)$, $26.80\left(\gamma \mathrm{CH}_{3}\right), 28.22\left[\mathrm{C}\left(\mathrm{CH}_{3}\right)_{3}\right], 43.36\left(\mathrm{CH}_{2}\right), 52.01,52.78$ $\left(\mathrm{OCH}_{3}\right), 80.16,80.72\left[\mathrm{OC}\left(\mathrm{CH}_{3}\right)_{3}\right], 130.40(\mathrm{C}), 130.53(\mathrm{C})$, $153.78(\mathrm{C}=\mathrm{O}), \quad 164.49 \quad(\mathrm{C}=\mathrm{O}) \quad \mathrm{ppm} . \quad \mathrm{m} / \mathrm{z}$ (HRESI-MS) $344.04692, \quad\left([\mathrm{M}+\mathrm{Na}]^{+}, \quad \mathrm{C}_{12} \mathrm{H}_{20} \mathrm{NNaO}_{4} \mathrm{Br}, \quad\right.$ requires 344.04734).

Synthesis of Boc-N(Et)-Z- $\triangle A b u(\beta-B r)-O M e \quad(Z-9 c)$ The general procedure described above was followed using Boc$Z-\triangle \mathrm{Abu}(\beta-\mathrm{Br})-\mathrm{OMe} \quad(Z-5 \mathbf{c}) \quad(0.147 \mathrm{~g}, 0.500 \mathrm{mmol})$ as reactant to afford $Z-9 \mathrm{c}(0.137 \mathrm{~g}, 85 \%)$ as a colorless oil that failed to crystallize. ${ }^{1} \mathrm{H}$ NMR $\left(400 \mathrm{MHz}, \mathrm{CDCl}_{3}\right)$ : (rotamers) $\delta=1.15,1.17\left(2 \mathrm{t}, J=7.2 \mathrm{~Hz}, J=7.2 \mathrm{~Hz}, 3 \mathrm{H}, \mathrm{CH}_{2} \mathrm{CH}_{3}\right)$, 1.39 (s, 9H, $\mathrm{CH}_{3}$ Boc), 2.79 (s, 3H, $\gamma \mathrm{CH}_{3}$ ), 3.40 (br. q, $J=$ $\left.7.2 \mathrm{~Hz}, 2 \mathrm{H}, \mathrm{CH}_{2} \mathrm{CH}_{3}\right), 3.75\left(\mathrm{~s}, 3 \mathrm{H}, \mathrm{CH}_{3} \mathrm{OMe}\right) \mathrm{ppm} .{ }^{13} \mathrm{C}$ NMR (100.6 MHz, $\mathrm{CDCl}_{3}$ ): (rotamers) $\delta=13.00,13.67$ $\left(\mathrm{CH}_{3}\right), 26.52\left(\gamma \mathrm{CH}_{3}\right), 27.84,28.16\left[\mathrm{C}\left(\mathrm{CH}_{3}\right)_{3}\right], 42.99,44.35$ $\left(\mathrm{CH}_{2}\right), 52.06,52.18\left(\mathrm{OCH}_{3}\right), 80.22,80.62\left[\mathrm{OC}\left(\mathrm{CH}_{3}\right)_{3}\right]$, $130.91,131.60$ (C), 140.10, 140.72 (C), 153.60, 154.15 $(\mathrm{C}=\mathrm{O}), 164.03,164.23 \quad(\mathrm{C}=\mathrm{O}) \mathrm{ppm} . \mathrm{m} / \mathrm{z}$ (HRESI-MS) $344.04679, \quad\left([\mathrm{M}+\mathrm{Na}]^{+}, \quad \mathrm{C}_{12} \mathrm{H}_{20} \mathrm{NNaO}_{4} \mathrm{Br}, \quad\right.$ requires 344.04734).

Synthesis of $\mathrm{Z}\left(\mathrm{NO}_{2}\right)-\mathrm{N}(\mathrm{Et})-\mathrm{Z}-\Delta \mathrm{Phe}(\beta-\mathrm{Br})-\mathrm{OMe}(\mathrm{Z}-10 \mathrm{a})$ The general procedure described above was followed using $\mathrm{Z}\left(\mathrm{NO}_{2}\right)-\mathrm{Z}-\Delta \mathrm{Phe}(\beta-\mathrm{Br})-\mathrm{OMe}(\mathrm{Z}-\mathbf{6 a})(0.131 \mathrm{~g}, 0.300 \mathrm{mmol})$ as reactant to afford Z-10a (Monteiro et al. 2011) (0.133 g, $96 \%)$

Synthesis of Z-N(Et)-Z- $\Delta P h e(\beta-B r)-O M e \quad(Z-10 b) \quad$ The general procedure described above was followed using $Z-Z$ $\triangle \mathrm{Abu}(\beta-\mathrm{Br})-\mathrm{OMe}(Z-6 \mathbf{b})(0.079 \mathrm{~g}, 0.203 \mathrm{mmol})$ as reactant to afford Z-10b (Monteiro et al. 2011) $(0.069 \mathrm{~g}, 82 \%)$.

Synthesis of Boc-N(Et)-Z-APhe( $\beta$-Br)-OMe (Z-10c) The general procedure described above was followed using Boc$Z-\Delta \mathrm{Phe}(\beta-\mathrm{Br})-\mathrm{OMe} \quad(Z-6 \mathbf{c}) \quad(0.178 \mathrm{~g}, \quad 0.500 \mathrm{mmol})$ as reactant to afford $Z-10 \mathrm{c}(0.170 \mathrm{~g}, 88 \%)$ as a light yellow oil. M.p. $59.5-60.0{ }^{\circ} \mathrm{C}$ (from ethyl acetate $/ n$-hexane). ${ }^{1} \mathrm{H}$ NMR $\left(400 \mathrm{MHz}, \mathrm{CDCl}_{3}\right)$ : (rotamers) $\delta=1.28(\mathrm{t}, J=7.6 \mathrm{~Hz}, 3 \mathrm{H}$, $\left.\mathrm{CH}_{2} \mathrm{CH}_{3}\right), 1.45,1.52\left(2 \mathrm{~s}, 9 \mathrm{H}, \mathrm{CH}_{3}\right.$ Boc $), 3.44,3.48(2 \mathrm{~s}, 3 \mathrm{H}$, $\mathrm{CH}_{3} \mathrm{OMe}$ ), 3.63 (q, $\left.J=7.5 \mathrm{~Hz}, 2 \mathrm{H}, \mathrm{CH}_{2} \mathrm{CH}_{3}\right), 7.32-7.36(\mathrm{~m}$, $5 \mathrm{H}, \mathrm{ArH}) \mathrm{ppm} .{ }^{13} \mathrm{C}$ NMR $\left(100.6 \mathrm{MHz}, \mathrm{CDCl}_{3}\right)$ : (rotamers) $\delta$ $=13.29,13.99\left(\mathrm{CH}_{3}\right), 28.16\left[\mathrm{C}\left(\mathrm{CH}_{3}\right)_{3}\right], 42.50,44.19\left(\mathrm{CH}_{2}\right)$, $51.95\left(\mathrm{OCH}_{3}\right), 80.83,81.17\left[\mathrm{OC}\left(\mathrm{CH}_{3}\right)_{3}\right], 128.16(\mathrm{CH})$, $128.22(\mathrm{CH}), 129.42(\mathrm{CH}), 132.29(\mathrm{C}), 135.66(\mathrm{C}), 139.18$ (C), $153.37(\mathrm{C}=\mathrm{O}), 164.66(\mathrm{C}=\mathrm{O})$ ppm. $\mathrm{C}_{17} \mathrm{H}_{22} \mathrm{NO}_{4} \mathrm{Br}$ (384.26): calcd. C 53.14, H 5.77, N 3.65; found C 53.29, H 5.77, N 3.86 . 
Synthesis of $Z\left(N_{2}\right)-N(E t)-Z-\Delta P h e(\beta-I)-O M e$ (Z-11a) The general procedure described above was followed using $\mathrm{Z}\left(\mathrm{NO}_{2}\right)-Z-\Delta \mathrm{Phe}(\beta-\mathrm{I})-\mathrm{OMe}(\mathrm{Z}-7 \mathbf{a})(0.121 \mathrm{~g}, 0.250 \mathrm{mmol})$ as reactant to afford $Z-11 a(0.121 \mathrm{~g}, 95 \%)$ as a light yellow solid. M.p. $134.0-135.0{ }^{\circ} \mathrm{C}$ (from ethyl acetate/n-hexane). ${ }^{1} \mathrm{H}$ NMR $\left(300 \mathrm{MHz}, \mathrm{CDCl}_{3}\right)$ : (rotamers) $\delta=1.35(\mathrm{t}, J=7.2 \mathrm{~Hz}$, $\left.3 \mathrm{H}, \mathrm{CH}_{2} \mathrm{CH}_{3}\right), 3.40,3.43$ (2s, 3H, $\left.\mathrm{CH}_{3} \mathrm{OMe}\right), 3.68-3.73$ (m, $\left.2 \mathrm{H}, \mathrm{CH}_{2} \mathrm{CH}_{3}\right), 5.30,5.35$ [2s, $\left.2 \mathrm{H}, \mathrm{CH}_{2} \mathrm{Z}\left(\mathrm{NO}_{2}\right)\right]$, 7.26-7.34 $(\mathrm{m}, 5 \mathrm{H}, \mathrm{ArH}), 7.53(\mathrm{~d}, J=8.8 \mathrm{~Hz}, 2 \mathrm{H}, \mathrm{ArH}), 8.21(\mathrm{~d}, J=$ $8.8 \mathrm{~Hz}, 2 \mathrm{H}, \mathrm{ArH}) \mathrm{ppm} .{ }^{13} \mathrm{C} \mathrm{NMR}\left(75.4 \mathrm{MHz}, \mathrm{CDCl}_{3}\right)$ : (rotamers) $\delta=13.15\left(\mathrm{CH}_{3}\right), 44.16,44.63\left(\mathrm{CH}_{2}\right), 52.25$, $53.68\left(\mathrm{OCH}_{3}\right), 66.18\left[\mathrm{CH}_{2} \mathrm{Z}\left(\mathrm{NO}_{2}\right)\right], 123.65,123.79(\mathrm{CH})$, $127.53,127.76(\mathrm{CH}), 127.93,128.06(\mathrm{CH}), 128.21(\mathrm{CH})$, 129.20, $129.36(\mathrm{CH}), 136.20(\mathrm{C}), 142.13(\mathrm{C}), 142.16(\mathrm{C})$, $143.71(\mathrm{C}), 147.51(\mathrm{C}), 153.71(\mathrm{C}=\mathrm{O}), 162.82(\mathrm{C}=\mathrm{O}) \mathrm{ppm}$. $\mathrm{C}_{20} \mathrm{H}_{19} \mathrm{~N}_{2} \mathrm{O}_{6} \mathrm{I}$ (510.28): calcd. C 47.08, H 3.75, N 5.49; found C 46.83, H 4.00, N 5.49.

Synthesis of Boc-N(Et)-Z-APhe( $\beta-I)-O M e \quad(Z-11 c)$ The general procedure described above was followed using Boc$Z-\Delta \mathrm{Phe}(\beta-\mathrm{I})-\mathrm{OMe}(Z-7 \mathrm{c})(0.133 \mathrm{~g}, 0.328 \mathrm{mmol})$ as reactant to afford $Z$-11c $(0.134 \mathrm{~g}, 95 \%)$ as a light orange oil. M.p. 69.5$70.0{ }^{\circ} \mathrm{C}$ (from diethyl ether $n$-hexane). ${ }^{1} \mathrm{H}$ NMR $(400 \mathrm{MHz}$, $\left.\mathrm{CDCl}_{3}\right)$ : (rotamers) $\delta=1.30\left(\mathrm{t}, J=7.2 \mathrm{~Hz}, 3 \mathrm{H}, \mathrm{CH}_{2} \mathrm{CH}_{3}\right)$, 1.48, 1.53 (2s, 9H, $\mathrm{CH}_{3}$ Boc), 3.46 (s, 3H, $\left.\mathrm{CH}_{3} \mathrm{OMe}\right), 3.61$ (q, $\left.J=7.2 \mathrm{~Hz}, 2 \mathrm{H}, \mathrm{CH}_{2} \mathrm{CH}_{3}\right), 7.27-7.35$ (m, 5H, ArH) ppm. ${ }^{13} \mathrm{C}$ NMR $\left(100.6 \mathrm{MHz}, \mathrm{CDCl}_{3}\right)$ : (rotamers) $\delta=13.36,14.08$ $\left(\mathrm{CH}_{3}\right), 28.24,28.35\left[\mathrm{C}\left(\mathrm{CH}_{3}\right)_{3}\right], 42.89,44.76\left(\mathrm{CH}_{2}\right), 51.97$ $\left(\mathrm{OCH}_{3}\right), 81.17\left[\mathrm{OC}\left(\mathrm{CH}_{3}\right)_{3}\right], 117.61(\mathrm{C}), 127.62(\mathrm{CH}), 127.97$, $128.10(\mathrm{CH}), 128.94(\mathrm{CH}), 137.16(\mathrm{C}), 142.72(\mathrm{C}), 153.25$ $(\mathrm{C}=\mathrm{O}), 163.18(\mathrm{C}=\mathrm{O})$ ppm. $\mathrm{C}_{17} \mathrm{H}_{22} \mathrm{NO}_{4} \mathrm{I}$ (431.27): calcd. $\mathrm{C}$ 47.34, H 5.14, N 3.25; found C 47.65, H 5.27, N 3.50.

Synthesis of the methyl esters of $N$-protected, $N$-ethyl dehydroamino acids

General procedure for the synthesis of the methyl esters of $\mathrm{N}$ protected, $N$-ethyl dehydroamino acids

The methyl ester of the $N$-protected dehydroamino acid was dissolved in anhydrous dichloromethane $\left(0.05 \mathrm{~mol} \mathrm{dm}^{-3}\right)$ followed by addition of 3.5 equiv. of potassium tertbutoxide and 2.5 equiv. of triethyloxonium tetrafluoroborate, under inert atmosphere. The reaction mixture was stirred at room temperature for 30 minutes. Then dichloromethane was added $\left(50 \mathrm{~cm}^{3}\right)$ and the organic phase was washed with $\mathrm{KHSO}_{4}\left(1 \mathrm{~mol} \mathrm{dm}^{-3}\right), \mathrm{NaHCO}_{3}$ $\left(1 \mathrm{~mol} \mathrm{dm}^{-3}\right)$ and brine (3 times $20 \mathrm{~cm}^{3}$ each), dried over $\mathrm{MgSO}_{4}$ and the solvent evaporated at reduced pressure.

Synthesis of $\mathrm{Z}\left(\mathrm{NO}_{2}\right)-\mathrm{N}(\mathrm{Et})-\triangle \mathrm{Ala}-\mathrm{OMe}$ (12a) The general procedure described above was followed using $\mathrm{Z}\left(\mathrm{NO}_{2}\right)$ $\triangle$ Ala-OMe (1a) $(0.211 \mathrm{~g}, 0.750 \mathrm{mmol})$ as reactant to afford $12 \mathbf{a}(0.204 \mathrm{~g}, 88 \%)$ as an orange oil that failed to crystallize. ${ }^{1} \mathrm{H}$ NMR $\left(400 \mathrm{MHz}, \mathrm{CDCl}_{3}\right): \delta=1.19(\mathrm{t}, J=6.9 \mathrm{~Hz}, 3 \mathrm{H}$, $\left.\mathrm{CH}_{2} \mathrm{CH}_{3}\right), 3.60\left(\mathrm{q}, J=6.9 \mathrm{~Hz}, 2 \mathrm{H}, \mathrm{CH}_{2} \mathrm{CH}_{3}\right), 3.72(\mathrm{~s}, 3 \mathrm{H}$, $\left.\mathrm{CH}_{3} \mathrm{OMe}\right), 5.21\left[\mathrm{~s}, 2 \mathrm{H}, \mathrm{CH}_{2} \mathrm{Z}\left(\mathrm{NO}_{2}\right)\right], 5.63\left(\mathrm{~s}, 1 \mathrm{H}, \beta_{\mathrm{CH}}\right)$, $6.19\left(\mathrm{~s}, 1 \mathrm{H}, \beta \mathrm{CH}_{2}\right), 7.46(\mathrm{~d}, J=8.4 \mathrm{~Hz}, 2 \mathrm{H}, \mathrm{ArH}), 8.20(\mathrm{~d}, J$ $=8.4 \mathrm{~Hz}, 2 \mathrm{H}, \mathrm{ArH}) \mathrm{ppm} .{ }^{13} \mathrm{C} \mathrm{NMR}\left(100.6 \mathrm{MHz} \mathrm{CDCl}_{3}\right): \delta$ $=13.39\left(\mathrm{CH}_{3}\right), 45.02\left(\mathrm{CH}_{2}\right), 52.44\left(\mathrm{OCH}_{3}\right), 65.94\left[\mathrm{CH}_{2}\right.$ $\left.\mathrm{Z}\left(\mathrm{NO}_{2}\right)\right], 121.61\left(\mathrm{CCH}_{2}\right), 123.67(\mathrm{CH}), 127.90(\mathrm{CH}), 138.68$ $(\alpha \mathrm{C}), 143.66(\mathrm{C}), 147.53(\mathrm{C}), 154.19(\mathrm{C}=\mathrm{O}), 164.66(\mathrm{C}=\mathrm{O})$ ppm. $\mathrm{C}_{14} \mathrm{H}_{16} \mathrm{~N}_{2} \mathrm{NaO}_{6}$ (308.29): calcd. C 54.54, H 5.23, N 9.09; found $\mathrm{C} 54.43, \mathrm{H} \mathrm{5.13,} \mathrm{N} \mathrm{9.11.} \mathrm{m/z} \mathrm{(HRESI-MS)}$ 331.09006, $\left([\mathrm{M}+\mathrm{Na}]^{+}, \mathrm{C}_{14} \mathrm{H}_{16} \mathrm{~N}_{2} \mathrm{NaO}_{6}\right.$, requires 331.09060).

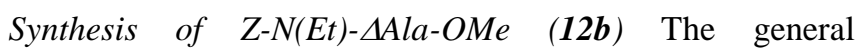
procedure described above was followed using Z- $\Delta$ AlaOMe (1b) $(0.118 \mathrm{~g}, 0.500 \mathrm{mmol})$ as reactant to afford $\mathbf{1 2 b}$ $(0.119 \mathrm{~g}, 91 \%)$ as a colorless oil that failed to crystallize. ${ }^{1} \mathrm{H}$
NMR $\left(300 \mathrm{MHz}, \mathrm{CDCl}_{3}\right)$ : (rotamers) $\delta=1.18(\mathrm{t}, J=6.9 \mathrm{~Hz}$, $3 \mathrm{H}, \mathrm{CH}_{2} \mathrm{CH}_{3}$ ), 3.60 (q, $J=6.9 \mathrm{~Hz}, 2 \mathrm{H}, \mathrm{CH}_{2} \mathrm{CH}_{3}$ ), 3.63, 3.87 $\left(2 \mathrm{~s}, 3 \mathrm{H}, \mathrm{CH}_{3} \mathrm{OMe}\right), 5.13\left(\mathrm{~s}, 2 \mathrm{H}, \mathrm{CH}_{2} \mathrm{Z}\right), 5.54\left(\mathrm{~s}, 1 \mathrm{H}, \beta_{\mathrm{CH}}\right)$, $6.09\left(\mathrm{~s}, 1 \mathrm{H}, \beta_{\mathrm{CH}}\right), 7.29-7.35(\mathrm{~m}, 5 \mathrm{H}, \mathrm{ArH}) \mathrm{ppm} .{ }^{13} \mathrm{C} \mathrm{NMR}$ (75.4 $\left.\mathrm{MHz}, \mathrm{CDCl}_{3}\right)$ : (rotamers) $\delta=13.43,13.93\left(\mathrm{CH}_{3}\right)$, $44.77\left(\mathrm{CH}_{2}\right), 52.21,52.94\left(\mathrm{OCH}_{3}\right), 66.45,67.39\left(\mathrm{CH}_{2} \mathrm{Z}\right)$, $120.06\left(\mathrm{BCH}_{2}\right), 127.85(\mathrm{CH}), 127.96(\mathrm{CH}), 128.36(\mathrm{CH})$, 136.18, $136.63(\mathrm{C}), 139.06(\alpha \mathrm{C}), 154.60(\mathrm{C}=\mathrm{O}), 164.90$ $(\mathrm{C}=\mathrm{O})$ ppm. $\mathrm{C}_{14} \mathrm{H}_{17} \mathrm{NO}_{4}$ (263.29): calcd. C 63.87, H 6.51, N 5.23; found $\mathrm{C} 63.30, \mathrm{H} 6.46, \mathrm{~N} 5.63 . \mathrm{m} / \mathrm{z}$ (HRESI-MS) 286.10537, ([M+Na] $]^{+}, \mathrm{C}_{14} \mathrm{H}_{17} \mathrm{NNaO}_{4}$, requires 286.10553).

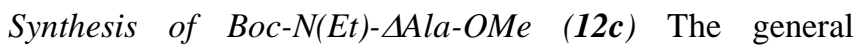
procedure described above was followed using Boc- $\Delta$ AlaOMe (1c) $(0.101 \mathrm{~g}, 0.500 \mathrm{mmol})$ as reactant to afford 12c $(0.090 \mathrm{~g}, 79 \%)$ as a colorless oil that failed to crystallize. ${ }^{1} \mathrm{H}$ NMR (300 MHz, $\left.\mathrm{CDCl}_{3}\right)$ : (rotamers) $\delta=1.17(\mathrm{t}, J=7.2 \mathrm{~Hz}$, $3 \mathrm{H}, \mathrm{CH}_{2} \mathrm{CH}_{3}$ ), 1.43 (s, 9H, $\mathrm{CH}_{3}$ Boc), 3.54 (br. q, $J=6.9$ $\left.\mathrm{Hz}, 2 \mathrm{H}, \mathrm{CH}_{2} \mathrm{CH}_{3}\right), 3.78,3.88\left(2 \mathrm{~s}, 3 \mathrm{H}, \mathrm{CH}_{3} \mathrm{OMe}\right), 5.40$ (s, $1 \mathrm{H}, \beta \mathrm{CH}), 5.91,5.93(2 \mathrm{~s}, 1 \mathrm{H}, \beta \mathrm{CH}) \mathrm{ppm} .{ }^{13} \mathrm{C} \mathrm{NMR}(75.4$ $\left.\mathrm{MHz}, \mathrm{CDCl}_{3}\right)$ : (rotamers) $\delta=13.47,14.09\left(\mathrm{CH}_{3}\right), 28.16$, $28.38\left[\mathrm{C}\left(\mathrm{CH}_{3}\right)_{3}\right], 44.32\left(\mathrm{CH}_{2}\right), 52.15,52.98\left(\mathrm{OCH}_{3}\right), 80.67$, $80.77\left[\mathrm{OC}\left(\mathrm{CH}_{3}\right)_{3}\right], 116.96,117.20\left(\beta^{2} \mathrm{CH}_{2}\right), 139.94(\alpha \mathrm{C})$, $161.14(\mathrm{C}=\mathrm{O}), 165.61 \quad(\mathrm{C}=\mathrm{O}) \quad \mathrm{ppm} . \quad \mathrm{m} / \mathrm{z} \quad$ (HRESI-MS) 252.12063, $\left([\mathrm{M}+\mathrm{Na}]^{+}, \mathrm{C}_{11} \mathrm{H}_{19} \mathrm{NNaO}_{4}\right.$, requires 252.12118).

Synthesis of Z $\mathrm{Z}\left(\mathrm{NO}_{2}\right)-\mathrm{N}(\mathrm{Et})-\mathrm{Z}-\triangle \mathrm{Abu}-\mathrm{OMe} \quad(\mathrm{Z}-13 \mathrm{a}) \quad$ The general procedure described above was followed using $\mathrm{Z}\left(\mathrm{NO}_{2}\right)-\mathrm{Z}-\Delta \mathrm{Abu}-\mathrm{OMe}(\mathrm{Z}-\mathbf{2 a}) \quad(0.221 \mathrm{~g}, 0.750 \mathrm{mmol})$ as reactant to afford $Z-13 a(0.217 \mathrm{~g}, 90 \%)$ as an orange oil that failed to crystallize. ${ }^{1} \mathrm{H} \mathrm{NMR}\left(400 \mathrm{MHz}, \mathrm{CDCl}_{3}\right)$ : (rotamers) $\delta=1.14\left(\mathrm{t}, J=7.2 \mathrm{~Hz}, 3 \mathrm{H}, \mathrm{CH}_{2} \mathrm{CH}_{3}\right), 1.81(\mathrm{~d}, J=7.2 \mathrm{~Hz}$, $3 \mathrm{H}, \gamma \mathrm{CH}_{3}$ ), 3.51 (br. q, $J=7.2 \mathrm{~Hz}, 2 \mathrm{H}, \mathrm{CH}_{2} \mathrm{CH}_{3}$ ), 3.73, 3.77 (2s, 3H, $\left.\mathrm{CH}_{3} \mathrm{OMe}\right), 5.19,5.30$ [br. s $+\mathrm{s}, 2 \mathrm{H}, \mathrm{CH}_{2} \mathrm{Z}\left(\mathrm{NO}_{2}\right)$ ], $7.00,7.04(2 \mathrm{q}, J=7.2 \mathrm{~Hz}$ and $J=7.2 \mathrm{~Hz}, 1 \mathrm{H}, \beta \mathrm{CH}), 7.42$, $7.55(2 \mathrm{~d}, J=8.8 \mathrm{~Hz}$ and $J=8.8 \mathrm{~Hz}, 2 \mathrm{H}, \mathrm{ArH}), 8.20,8.24$ $(2 \mathrm{~d}, J=8.8 \mathrm{~Hz}$ and $J=8.8 \mathrm{~Hz}, 2 \mathrm{H}, \mathrm{ArH}) \mathrm{ppm} .{ }^{13} \mathrm{C} \mathrm{NMR}$ $\left(100.6 \mathrm{MHz}, \mathrm{CDCl}_{3}\right)$ : (rotamers) $\delta=12.94\left(\mathrm{CH}_{3}\right), 13.76$, $13.91\left(\gamma \mathrm{CH}_{3}\right), 44.08\left(\mathrm{CH}_{2}\right), 52.20\left(\mathrm{OCH}_{3}\right), 65.68,65.89$ $\left[\mathrm{CH}_{2} \mathrm{Z}\left(\mathrm{NO}_{2}\right)\right], 123.64,123.77(\mathrm{CH}), 127.73,127.92(\mathrm{CH})$, $131.59,131.96(\mathrm{C}), 138.40,139.07(\beta \mathrm{CH}), 144.00,144.09$ (C), $147.46(\mathrm{C}), 154.56,154.75(\mathrm{C}=\mathrm{O}), 164.54,164.87$ $(\mathrm{C}=\mathrm{O})$ ppm. $\mathrm{C}_{15} \mathrm{H}_{18} \mathrm{~N}_{2} \mathrm{O}_{6}$ (322.31): calcd. C 55.90, H 5.63, N 8.69; found $\mathrm{C} 55.82, \mathrm{H} \mathrm{5.41,} \mathrm{N} \mathrm{8.65.} \mathrm{m/z} \mathrm{(HRESI-MS)}$ 323.12432, $\left([\mathrm{M}+\mathrm{H}]^{+}, \mathrm{C}_{15} \mathrm{H}_{19} \mathrm{~N}_{2} \mathrm{O}_{6}\right.$, requires 323.12431).

Synthesis of Z-N(Et)-Z- $\triangle A b u-O M e$ (Z-13b) The general procedure described above was followed using Z-Z- $\triangle$ AbuOMe $(Z-2 b)(0.062 \mathrm{~g}, 0.250 \mathrm{mmol})$ as reactant to afford $Z$ 13b $(0.064 \mathrm{~g}, 93 \%)$ as a colorless oil that failed to crystallize. ${ }^{1} \mathrm{H}$ NMR $\left(400 \mathrm{MHz}, \mathrm{CDCl}_{3}\right.$ ): (rotamers) $\delta=1.13$ $\left(\mathrm{t}, J=7.2 \mathrm{~Hz}, 3 \mathrm{H}, \mathrm{CH}_{2} \mathrm{CH}_{3}\right), 1.76,1.80(2 \mathrm{~d}, J=7.2 \mathrm{~Hz}$ and $\left.J=7.2 \mathrm{~Hz}, 3 \mathrm{H}, \gamma \mathrm{CH}_{3}\right), 3.46-3.50\left(\mathrm{~m}, 2 \mathrm{H}, \mathrm{CH}_{2} \mathrm{CH}_{3}\right), 3.66$, $3.77\left(2 \mathrm{~s}, 3 \mathrm{H}, \mathrm{CH}_{3} \mathrm{OMe}\right), 5.11,5.21\left(2 \mathrm{~s}, 2 \mathrm{H}, \mathrm{CH}_{2} \mathrm{Z}\right), 6.93$, $7.02(2 \mathrm{q}, J=7.2 \mathrm{~Hz}$ and $J=7.2 \mathrm{~Hz}, 1 \mathrm{H}, \beta \mathrm{CH}), 7.28-7.39$ $(\mathrm{m}, 5 \mathrm{H}, \mathrm{ArH}) \mathrm{ppm} .{ }^{13} \mathrm{C} \mathrm{NMR}\left(100.6 \mathrm{MHz}, \mathrm{CDCl}_{3}\right)$ : (rotamers) $\delta=13.00\left(\mathrm{CH}_{3}\right), 13.83,13.92\left(\gamma \mathrm{CH}_{3}\right), 43.80$, $43.91\left(\mathrm{CH}_{2}\right), 52.02,52.11\left(\mathrm{OCH}_{3}\right), 67.10,67.36\left(\mathrm{CH}_{2} \mathrm{Z}\right)$, 127.66, $127.76(\mathrm{CH}), 127.82,127.96(\mathrm{CH}), 128.33,128.46$ $(\mathrm{CH}), 131.77,132.19$ (C), 139.30, 136.66 (C), 138.01, $138.81(\beta C H), 155.01,155.27(\mathrm{C}=\mathrm{O}), 164.82,165.11(\mathrm{C}=\mathrm{O})$ ppm. $\mathrm{m} / \mathrm{z}$ (HRESI-MS) 300.12063, $\left([\mathrm{M}+\mathrm{Na}]^{+}\right.$, $\mathrm{C}_{15} \mathrm{H}_{19} \mathrm{NNaO}_{4}$, requires 300.12118).

Synthesis of Boc-N(Et)-Z- $\triangle A b u-O M e(Z-13 c)$ The general procedure described above was followed using Boc-Z$\triangle$ Abu-OMe (Z-2c) $(0.294 \mathrm{~g}, 1.364 \mathrm{mmol})$ as reactant to afford $Z$-13c $(0.304 \mathrm{~g}, 92 \%)$ as a colorless oil that failed to crystallize. ${ }^{1} \mathrm{H}$ NMR (400 MHz, $\mathrm{CDCl}_{3}$ ): (rotamers) $\delta=1.06$ 
(t, $\left.J=7.2 \mathrm{~Hz}, 3 \mathrm{H}, \mathrm{CH}_{2} \mathrm{CH}_{3}\right), 1.35,1.46\left(2 \mathrm{~s}, 9 \mathrm{H}, \mathrm{CH}_{3}\right.$ Boc), $1.75\left(\mathrm{~d}, J=7.2 \mathrm{~Hz}, 3 \mathrm{H}, \gamma \mathrm{CH}_{3}\right), 3.32,3.40(2 \mathrm{q}, J=7.2 \mathrm{~Hz}$ and $\left.J=7.2 \mathrm{~Hz}, 2 \mathrm{H}, \mathrm{CH}_{2} \mathrm{CH}_{3}\right), 3.71,3.72\left(2 \mathrm{~s}, 3 \mathrm{H}, \mathrm{CH}_{3}\right.$ $\mathrm{OMe}), 6.79,6.89(2 \mathrm{q}, J=7.2 \mathrm{~Hz}$ and $J=7.2 \mathrm{~Hz}, 1 \mathrm{H}, \beta \mathrm{CH})$ ppm. ${ }^{13} \mathrm{C} \mathrm{NMR}\left(100.6 \mathrm{MHz}, \mathrm{CDCl}_{3}\right)$ : (rotamers) $\delta=12.99$, $13.02\left(\mathrm{CH}_{3}\right), 13.68,13.81\left(\gamma \mathrm{CH}_{3}\right), 28.13,28.25\left[\mathrm{C}\left(\mathrm{CH}_{3}\right)_{3}\right]$, 42.84, $43.99\left(\mathrm{CH}_{2}\right)$, 51.83, 51.88 $\left(\mathrm{OCH}_{3}\right), 79.80,80.08$ $\left[\mathrm{OC}\left(\mathrm{CH}_{3}\right)_{3}\right], 132.42,132.54(\alpha \mathrm{C}), 135.93,136.17(\beta \mathrm{CH})$, $154.17, \quad 154.38 \quad(\mathrm{C}=\mathrm{O}), \quad 165.01,165.45 \quad(\mathrm{C}=\mathrm{O})$ ppm. $\mathrm{C}_{12} \mathrm{H}_{21} \mathrm{NO}_{4}$ (243.30): calcd. C 59.24, H 8.70, N 5.76; found $\mathrm{C} 59.02, \mathrm{H}$ 8.70, $\mathrm{N}$ 5.71. $\mathrm{m} / \mathrm{z}$ (HRESI-MS) 266.13655, $\left([\mathrm{M}+\mathrm{Na}]^{+}, \mathrm{C}_{12} \mathrm{H}_{21} \mathrm{NNaO}_{4}\right.$, requires 266.13683).

Synthesis of Tos-N(Et)-Z- $\triangle A b u-O M e$ (Z-13d) The general procedure described above was followed using Tos- $Z$ $\triangle \mathrm{Abu}-\mathrm{OMe}(Z-2 d)(0.135 \mathrm{~g}, 0.500 \mathrm{mmol})$ as reactant to afford $Z$-13d $(0.122 \mathrm{~g}, 82 \%)$ as a colorless oil that failed to crystallize. ${ }^{1} \mathrm{H}$ NMR $\left(400 \mathrm{MHz}, \mathrm{CDCl}_{3}\right): \delta=1.10(\mathrm{t}, J=7.2$ $\left.\mathrm{Hz}, 3 \mathrm{H}, \mathrm{CH}_{2} \mathrm{CH}_{3}\right), 1.98\left(\mathrm{~d}, J=7.2 \mathrm{~Hz}, 3 \mathrm{H}, \gamma \mathrm{CH}_{3}\right), 2.42$ (s, $3 \mathrm{H}, \mathrm{CH}_{3}$ Tos), $3.21,3.64$ (2 br. s, $2 \mathrm{H}, \mathrm{CH}_{2} \mathrm{CH}_{3}$ ), 3.51 (s, $3 \mathrm{H}$, $\left.\mathrm{CH}_{3} \mathrm{OMe}\right), 7.28(\mathrm{~d}, J=8.4 \mathrm{~Hz}, 2 \mathrm{H}, \mathrm{ArH}), 7.32$ (q, $J=7.2$ $\mathrm{Hz}, 1 \mathrm{H}, \beta \mathrm{CH}), 7.70(\mathrm{~d}, J=8.4 \mathrm{~Hz}, 2 \mathrm{H}, \mathrm{ArH}) \mathrm{ppm} .{ }^{13} \mathrm{C}$ NMR $\left(100.6 \mathrm{MHz}, \mathrm{CDCl}_{3}\right): \delta=14.01\left(\mathrm{CH}_{3}\right), 15.22\left(\gamma \mathrm{CH}_{3}\right)$, $21.49\left(\mathrm{Tos} \mathrm{CH}_{3}\right), 44.06\left(\mathrm{CH}_{2}\right), 51.76\left(\mathrm{OCH}_{3}\right), 127.66(\mathrm{CH})$, $128.72(\mathrm{C}), 129.18(\mathrm{CH}), 136.99(\mathrm{C}), 143.10(\mathrm{C}), 146.47$ $(\mathrm{CH}), 164.39(\mathrm{C}=\mathrm{O}) \mathrm{ppm} . \mathrm{m} / \mathrm{z}$ (HRESI-MS) 320.09270, $\left([\mathrm{M}+\mathrm{Na}]^{+}, \mathrm{C}_{14} \mathrm{H}_{19} \mathrm{NNaO}{ }_{4} \mathrm{~S}\right.$, requires 320.09325$)$.

Synthesis of $\mathrm{Z}\left(\mathrm{NO}_{2}\right)-\mathrm{N}(\mathrm{Et})-\mathrm{Z}-\Delta P h e-O M e \quad(\mathrm{Z}-14 a)$ The general procedure described above was followed using $\mathrm{Z}\left(\mathrm{NO}_{2}\right)-\mathrm{Z}-\Delta \mathrm{Phe}-\mathrm{OMe}(\mathrm{Z}-3 \mathbf{a}) \quad(0.270 \mathrm{~g}, 0.758 \mathrm{mmol})$ as reactant to afford $Z-14 a(0.283 \mathrm{~g}, 97 \%)$ as a light yellow oil that failed to crystallize. ${ }^{1} \mathrm{H}$ NMR (400 $\left.\mathrm{MHz}, \mathrm{CDCl}_{3}\right)$ : (rotamers) $\delta=1.10,1.21(2 \mathrm{t}, J=7.2 \mathrm{~Hz}$ and $J=7.2 \mathrm{~Hz}, 3 \mathrm{H}$, $\mathrm{CH}_{2} \mathrm{CH}_{3}$ ), 3.29, 3.47, 3.72 (3br. s, $2 \mathrm{H}, \mathrm{CH}_{2} \mathrm{CH}_{3}$ ), 3.79, 3.85 $\left(2 \mathrm{~s}, 3 \mathrm{H}, \mathrm{CH}_{3} \mathrm{OMe}\right), 5.21,5.37$ [br. s $+\mathrm{s}, 2 \mathrm{H}, \mathrm{CH}_{2} \mathrm{Z}\left(\mathrm{NO}_{2}\right)$ ], 7.34-7.58 (m, 8H, ArH + $\beta \mathrm{CH}), 8.12,8.26[2 \mathrm{~d}, J=8.8 \mathrm{~Hz}$ and $\left.J=8.8 \mathrm{~Hz}, 2 \mathrm{H}, \mathrm{Z}\left(\mathrm{NO}_{2}\right)\right] \mathrm{ppm} .{ }^{13} \mathrm{C} \mathrm{NMR}(100.6 \mathrm{MHz}$, $\left.\mathrm{CDCl}_{3}\right)$ : (rotamers) $\delta=12.76,13.57\left(\mathrm{CH}_{3}\right), 43.83,44.16$ $\left(\mathrm{CH}_{2}\right), 52.53,52.55\left(\mathrm{OCH}_{3}\right), 65.90,66.02\left[\mathrm{CH}_{2} \mathrm{Z}\left(\mathrm{NO}_{2}\right)\right]$, 123.57, $123.78(\mathrm{CH}), 127.89,127.99(\mathrm{CH}), 128.73,128.92$ $(\mathrm{CH}), 129.78(\mathrm{C}), 130.04,130.09(\mathrm{CH}), 130.43(\mathrm{CH})$, 132.58, 132.78 (C), 136.43, $136.81(\mathrm{CH}), 143.83,144.01$ (C), 147.45, 147.62 (C), 154.94, $155.15(\mathrm{C}=\mathrm{O}), 165.52$, $166.01(\mathrm{C}=\mathrm{O}) \mathrm{ppm} . \mathrm{m} / \mathrm{z}$ (HRESI-MS) 385.13941, $\left([\mathrm{M}+\mathrm{H}]^{+}\right.$, $\mathrm{C}_{20} \mathrm{H}_{21} \mathrm{~N}_{2} \mathrm{O}_{6}$, requires 385.13996).

Synthesis of Z-N(Et)-Z-APhe-OMe (Z-14b) The general procedure described above was followed using $Z-Z-\Delta P h e-$ OMe (Z-3b) (0.749 g, $2.405 \mathrm{mmol})$ as reactant to afford $Z$ 14b $(0.733 \mathrm{~g}, 90 \%)$ as a yellow oil that failed to crystallize. ${ }^{1} \mathrm{H}$ NMR (400 MHz, $\mathrm{CDCl}_{3}$ ): (rotamers) $\delta=1.07(\mathrm{t}, J=7.2$ $\mathrm{Hz}, 3 \mathrm{H}, \mathrm{CH}_{2} \mathrm{CH}_{3}$ ), 3.24, 3.40 (2br. s, $2 \mathrm{H}, \mathrm{CH}_{2} \mathrm{CH}_{3}$ ), 3.68, 3.84 (s, 3H, $\mathrm{CH}_{3} \mathrm{OMe}$ ), 5.17, 5.28 (br. s + s, $2 \mathrm{H}, \mathrm{CH}_{2} \mathrm{Z}$ ), 7.23-7.52 (m, 10H, ArH + $\beta \mathrm{CH}), 7.59(\mathrm{~s}, 1 \mathrm{H}, \mathrm{ArH}) \mathrm{ppm}$. ${ }^{13} \mathrm{C} \mathrm{NMR}\left(100.6 \mathrm{MHz}, \mathrm{CDCl}_{3}\right)$ : (rotamers) $\delta=12.84,13.58$ $\left(\mathrm{CH}_{3}\right), 43.55,44.00\left(\mathrm{CH}_{2}\right), 52.33,52.47\left(\mathrm{OCH}_{3}\right), 67.37$, $67.50\left(\mathrm{CH}_{2} \mathrm{Z}\right), 127.81(\mathrm{CH}), 127.86(\mathrm{CH}), 128.32,128.50$ $(\mathrm{CH}), 128.67,128.78(\mathrm{CH}), 129.86(\mathrm{CH}), 130.01(\mathrm{CH})$, $130.06(\mathrm{C}), 132.84,132.94(\mathrm{C}), 135.66(\mathrm{CH}), 136.44$, $136.66(\mathrm{C}), 155.52,155.57(\mathrm{C}=\mathrm{O}), 165.81,166.24(\mathrm{C}=\mathrm{O})$ ppm. $\quad \mathrm{m} / \mathrm{z}$ (HRESI-MS) 362.13628, ([M+Na $]^{+}$, $\mathrm{C}_{20} \mathrm{H}_{21} \mathrm{NNaO}_{4}$, requires 362.13683).

Synthesis of Boc-N(Et)-Z-APhe-OMe (Z-14c) The general procedure described above was followed using Boc- $Z-\Delta P$ PeOMe $(Z-3 c)(0.206 \mathrm{~g}, 0.750 \mathrm{mmol})$ as reactant to afford $Z$ 14c $(0.208 \mathrm{~g}, 92 \%)$ as a light yellow oil that failed to crystallize. ${ }^{1} \mathrm{H}$ NMR (400 MHz, $\mathrm{CDCl}_{3}$ ): (rotamers) $\delta=1.04$ (t, $\left.J=7.2 \mathrm{~Hz}, 3 \mathrm{H}, \mathrm{CH}_{2} \mathrm{CH}_{3}\right), 1.40,1.54\left(2 \mathrm{~s}, 9 \mathrm{H}, \mathrm{CH}_{3} \mathrm{Boc}\right)$, $3.10,3.30,3.67$ (3br. s, $2 \mathrm{H}, \mathrm{CH}_{2} \mathrm{CH}_{3}$ ), 3.83 (s, $3 \mathrm{H}, \mathrm{CH}_{3}$ $\mathrm{OMe})$, 7.36-7.39 (m, 4H, ArH + $\beta \mathrm{CH})$ 7.50-7.53 (m, $2 \mathrm{H}$, ArH) ppm. $\left.{ }^{13} \mathrm{C} \mathrm{NMR} \mathrm{(100.6} \mathrm{MHz,} \mathrm{CDCl}_{3}\right)$ : (rotamers) $\delta=$ 12.94, $13.54\left(\mathrm{CH}_{3}\right), 28.17,28.37\left[\mathrm{C}\left(\mathrm{CH}_{3}\right)_{3}\right], 42.53,44.11$ $\left(\mathrm{CH}_{2}\right)$, 52.24, $52.31\left(\mathrm{OCH}_{3}\right), 80.44,80.60\left[\mathrm{OC}\left(\mathrm{CH}_{3}\right)_{3}\right]$, $128.57,128.71(\mathrm{CH}), 129.63,129.73(\mathrm{CH}), 129.87,129.95$ $(\mathrm{CH}), 130.68(\mathrm{C}), 133.23,133.28(\mathrm{C}), 133.99(\mathrm{CH}), 154.56$, $154.78(\mathrm{C}=\mathrm{O}), 166.11,166.68(\mathrm{C}=\mathrm{O}) \mathrm{ppm} . \mathrm{m} / \mathrm{z}$ (HRESIMS $) 328.15193, \quad\left([\mathrm{M}+\mathrm{Na}]^{+}, \quad \mathrm{C}_{17} \mathrm{H}_{23} \mathrm{NNaO}_{4}, \quad\right.$ requires 328.15248).

Synthesis of Tos-N(Et)-Z-APhe-OMe (Z-14d) The general procedure described above was followed using Tos-Z- $\Delta$ PheOMe $(Z-3 d)(0.166 \mathrm{~g}, 0.500 \mathrm{mmol})$ as reactant to afford $Z$ 14d $(0.165 \mathrm{~g}, 92 \%)$ as a light yellow oil. M.p. 111.0-112.0 ${ }^{\circ} \mathrm{C}$ (from diethyl ether/petroleum ether). ${ }^{1} \mathrm{H}$ NMR (300 $\mathrm{MHz}, \mathrm{CDCl}_{3}$ ): $\delta=1.03\left(\mathrm{t}, J=7.2 \mathrm{~Hz}, 3 \mathrm{H}, \mathrm{CH}_{2} \mathrm{CH}_{3}\right), 2.44$ (s, $3 \mathrm{H}, \mathrm{CH}_{3}$ Tos), 3.49 (br. s, $2 \mathrm{H}, \mathrm{CH}_{2} \mathrm{CH}_{3}$ ), 3.58 (s, $3 \mathrm{H}$, $\left.\mathrm{CH}_{3} \mathrm{OMe}\right), 7.29$ (d, $\left.J=8.1 \mathrm{~Hz}, 2 \mathrm{H}, \mathrm{ArH}\right), 7.39-7.41$ (m, $3 \mathrm{H}, \mathrm{ArH}), 7.75$ (d, $J=8.1 \mathrm{~Hz}, 2 \mathrm{H}, \mathrm{ArH}), 7.84-7.89$ (m, 3H, $\mathrm{ArH}+\beta \mathrm{CH}) \mathrm{ppm} .{ }^{13} \mathrm{C} \mathrm{NMR}\left(75.4 \mathrm{MHz}, \mathrm{CDCl}_{3}\right): \delta=13.38$ $\left(\mathrm{CH}_{2} \mathrm{CH}_{3}\right), 21.53\left(\mathrm{Tos} \mathrm{CH}_{3}\right), 44.13\left(\mathrm{CH}_{2}\right), 52.08\left(\mathrm{OCH}_{3}\right)$, $125.42(\mathrm{C}), 128.04(\mathrm{CH}), 128.62(\mathrm{CH}), 129.17(\mathrm{CH}), 130.80$ $(\mathrm{CH}), 131.15(\mathrm{CH}), 132.51(\mathrm{C}), 136.62(\mathrm{C}), 143.22(\mathrm{C})$, $143.32(\mathrm{CH}), 165.76(\mathrm{C}=\mathrm{O})$ ppm. $\mathrm{C}_{19} \mathrm{H}_{21} \mathrm{NO}_{4} \mathrm{~S}$ (359.44): calcd. C 63.49, H 5.89, N 3.90, S 8.92; found C 63.55, H 5.97, N 3.96, S 8.73.

Synthesis of $N$-protected, $N$-ethyl dehydroamino acids

General procedure for the synthesis of N-protected, N-ethyl dehydroamino acids

The methyl ester of the $N$-protected, $N$-ethyl dehydroamino acid was dissolved in dioxane $\left(0.2 \mathrm{~mol} \mathrm{dm}^{-3}\right)$, followed by addition of $3 \mathrm{~cm}^{3}$ of $\mathrm{NaOH}\left(1 \mathrm{~mol} \mathrm{dm} \mathrm{dm}^{-3}\right)$. The solution was stirred at room temperature for 2 hours and then acidified to pH 2-3 with $\mathrm{KHSO}_{4}\left(1 \mathrm{~mol} \mathrm{dm}{ }^{-3}\right)$ and extracted with ethyl acetate $\left(5 \times 10 \mathrm{~cm}^{3}\right)$. The organic extracts were collected, dried over $\mathrm{MgSO}_{4}$ and evaporated at reduced pressure.

Synthesis of Boc-N(Et)- $\triangle A l a-O H \quad(15 c)$ The general procedure described above was followed using Boc- $N(\mathrm{Et})$ $\triangle$ Ala-OMe (12c) $(0.134 \mathrm{~g}, 0.587 \mathrm{mmol})$ as reactant to afford 15c $(0.115 \mathrm{~g}, 91 \%)$ as a light yellow oil that failed to crystallize. ${ }^{1} \mathrm{H}$ NMR $\left(400 \mathrm{MHz}, \mathrm{CDCl}_{3}\right): \delta=1.18(\mathrm{t}, J=7.2$ $\mathrm{Hz}, 3 \mathrm{H}, \mathrm{CH}_{2} \mathrm{CH}_{3}$ ), 1.44 (s, 9H, $\mathrm{CH}_{3}$ Boc), 3.56 (q, $J=6.9$ $\left.\mathrm{Hz}, 2 \mathrm{H}, \mathrm{CH}_{2} \mathrm{CH}_{3}\right), 5.53(\mathrm{~s}, 1 \mathrm{H}, \beta \mathrm{CH}), 6.07(\mathrm{~s}, 1 \mathrm{H}, \beta \mathrm{CH})$ ppm. ${ }^{13} \mathrm{C}$ NMR $\left(100.6 \mathrm{MHz}, \mathrm{CDCl}_{3}\right): \delta=15.10\left(\mathrm{CH}_{3}\right)$, $28.36\left[\mathrm{C}\left(\mathrm{CH}_{3}\right)_{3}\right], 44.29\left(\mathrm{CH}_{2}\right), 81.25\left[\mathrm{OC}\left(\mathrm{CH}_{3}\right)_{3}\right], 118.90$ $\left(\beta \mathrm{CH}_{2}\right), 139.53(\alpha \mathrm{C}), 156.33(\mathrm{C}=\mathrm{O}), 169.13(\mathrm{C}=\mathrm{O}) \mathrm{ppm}$. $\mathrm{m} / \mathrm{z}$ (HRESI-MS) 216.12374, $\left([\mathrm{M}+\mathrm{H}]^{+}, \mathrm{C}_{10} \mathrm{H}_{17} \mathrm{NO}_{4}\right.$, requires 216.12356).

Synthesis of Z-N(Et)-Z- $\triangle A b u-O H$ (Z-16b) The general procedure described above was followed using $Z-N(E t)-Z$ $\triangle$ Abu-OMe $(Z-13 b)(0.277 \mathrm{~g}, 1.000 \mathrm{mmol})$ as reactant to afford Z-16b $(0.249 \mathrm{~g}, 95 \%)$ as a yellow oil that failed to crystallize. ${ }^{1} \mathrm{H}$ NMR $\left(400 \mathrm{MHz}, \mathrm{CDCl}_{3}\right)$ : (rotamers) $\delta=1.15$ $\left(\mathrm{t}, J=7.2 \mathrm{~Hz}, 3 \mathrm{H}, \mathrm{CH}_{2} \mathrm{CH}_{3}\right), 1.79\left(\mathrm{~d}, J=7.2 \mathrm{~Hz}, 3 \mathrm{H}, \gamma \mathrm{CH}_{3}\right)$, 3.50 (q, $J=7.2 \mathrm{~Hz}, 2 \mathrm{H}, \mathrm{CH}_{2} \mathrm{CH}_{3}$ ), 5.11 (br. s, $2 \mathrm{H}, \mathrm{CH}_{2} \mathrm{Z}$ ), $7.08(\mathrm{q}, J=7.2 \mathrm{~Hz}, 1 \mathrm{H}, \beta \mathrm{CH}), 7.25-7.40(\mathrm{~m}, 5 \mathrm{H}, \mathrm{ArH}) \mathrm{ppm}$. ${ }^{13} \mathrm{C}$ NMR $\left(100.6 \mathrm{MHz}, \mathrm{CDCl}_{3}\right): \delta=13.03\left(\mathrm{CH}_{3}\right), 14.10$ $\left(\gamma \mathrm{CH}_{3}\right), 43.75\left(\mathrm{CH}_{2}\right), 67.27\left(\mathrm{CH}_{2} \mathrm{Z}\right), 127.57(\mathrm{CH}), 127.81$ $(\mathrm{CH}), 128.34(\mathrm{CH}), 131.28(\mathrm{C}), 136.53(\mathrm{C}), 140.67(\beta \mathrm{CH})$, $155.24(\mathrm{C}=\mathrm{O}), \quad 169.36 \quad(\mathrm{C}=\mathrm{O}) \quad \mathrm{ppm} . \quad \mathrm{m} / \mathrm{z} \quad$ (HRESI-MS) 264.12332, $\left([\mathrm{M}+\mathrm{H}]^{+}, \mathrm{C}_{14} \mathrm{H}_{17} \mathrm{NO}_{4}\right.$, requires 264.12358). 
Synthesis of Z-N(Et)-Z- $\triangle P h e-O H \quad(Z-17 b)$ The general procedure described above was followed using Z-N(Et)-Z$\Delta$ Phe-OMe $(Z-14 b)(0.339 \mathrm{~g}, 1.000 \mathrm{mmol})$ as reactant to afford $Z$-17b $(0.307 \mathrm{~g}, 94 \%)$ as a light yellow oil that failed to crystallize. ${ }^{1} \mathrm{H} \mathrm{NMR}\left(400 \mathrm{MHz}, \mathrm{CDCl}_{3}\right.$ ): (rotamers) $\delta=$ $1.11\left(\mathrm{t}, J=7.2 \mathrm{~Hz}, 3 \mathrm{H}, \mathrm{CH}_{2} \mathrm{CH}_{3}\right), 3.26,3.46$ (2br. s, $2 \mathrm{H}$, $\mathrm{CH}_{2} \mathrm{CH}_{3}$ ), 5.16 (s, 2H, $\left.\mathrm{CH}_{2} \mathrm{Z}\right), 7.23-7.25$ (m, 3H, ArH), 7.36-7.41 (m, 4H, ArH), 7.52-7.56 (m, 3H, ArH) $7.64(\mathrm{~s}$, $1 \mathrm{H}, \beta \mathrm{CH}) \mathrm{ppm} .{ }^{13} \mathrm{C}$ NMR $\left(100.6 \mathrm{MHz}, \mathrm{CDCl}_{3}\right): \delta=12.89$ $\left(\mathrm{CH}_{3}\right), 43.58\left(\mathrm{CH}_{2}\right), 67.54\left(\mathrm{CH}_{2} \mathrm{Z}\right), 127.62(\mathrm{CH}), 127.79$ $(\mathrm{CH}), 128.30(\mathrm{CH}), 128.40(\mathrm{C}), 128.77(\mathrm{CH}), 128.87(\mathrm{CH})$, $130.27(\mathrm{CH}), 132.57(\mathrm{C}), 136.32(\mathrm{C}), 137.75(\mathrm{CH}), 155.52$ $(\mathrm{C}=\mathrm{O}), 170.63(\mathrm{C}=\mathrm{O}) \mathrm{ppm} . \mathrm{m} / \mathrm{z}$ (HRESI-MS) 326.13868, $\left([\mathrm{M}+\mathrm{H}]^{+}, \mathrm{C}_{19} \mathrm{H}_{19} \mathrm{NO}_{4}\right.$, requires 326.13923).

Synthesis of the methyl esters of $N$-protected, $N$-ethyl dehydrodipeptides

General procedure for the synthesis of the methyl esters of $N$ protected, $N$-ethyl dehydrodipeptides

The $N$-protected, $N$-ethyl dehydroamino acid was dissolved in acetonitrile $\left(0.05 \mathrm{~mol} \mathrm{dm}^{-3}\right)$, followed by addition of 1.0 equiv. of HOBt, 1.1 equiv. of DCC, 1.0 equiv. of HCl.H-LPhe-OMe and 1.0 equiv. of $\mathrm{NEt}_{3}$ in an ice bath. After stirring for 16 hours at room temperature, the mixture was filtered and the solvent was evaporated at reduced pressure. The residue was dissolved in ethyl acetate $\left(100 \mathrm{~cm}^{3}\right)$ and washed with $\mathrm{KHSO}_{4}\left(1 \mathrm{~mol} \mathrm{dm}{ }^{-3}\right), \mathrm{NaHCO}_{3}\left(1 \mathrm{~mol} \mathrm{dm}^{-3}\right)$ and brine $\left(3 \times 30 \mathrm{~cm}^{3}\right.$ each). The organic layer was dried with $\mathrm{MgSO}_{4}$ and the solvent evaporated at reduced pressure.

Synthesis of Z-N(Et)-Z- $\triangle A b u-L-P h e-O M e(18 b)$ The general procedure described above was followed using Z-N(Et)-Z$\triangle \mathrm{Abu}-\mathrm{OH}(\mathrm{Z}-\mathbf{1 6 b})(0.165 \mathrm{~g}, 0.629 \mathrm{mmol})$ as reactant to afford $18 \mathbf{b}(0.233 \mathrm{~g}, 87 \%)$ as a colorless oil that failed to crystallize. ${ }^{1} \mathrm{H}$ NMR $\left(300 \mathrm{MHz}, \mathrm{CDCl}_{3}\right): \delta=1.02$ (br. s, $\left.3 \mathrm{H}, \mathrm{CH}_{2} \mathrm{CH}_{3}\right), 1.62\left(\mathrm{~d}, J=7.2 \mathrm{~Hz}, 3 \mathrm{H}, \gamma \mathrm{CH}_{3}\right), 3.07-3.40(\mathrm{~m}$, $\left.4 \mathrm{H}, \mathrm{CH}_{2} \mathrm{CH}_{3}+\mathrm{\beta CH}_{2}\right), 3.71\left(\mathrm{~s}, 3 \mathrm{H}, \mathrm{CH}_{3} \mathrm{OMe}\right), 4.85-5.20$ $\left(\mathrm{m}, 3 \mathrm{H}, \mathrm{CH}_{2} \mathrm{Z}+\alpha \mathrm{CH}\right), 6.33(\mathrm{~d}, J=7.2 \mathrm{~Hz}, 1 \mathrm{H}, \mathrm{NH}), 6.87$ (q, $J=7.2 \mathrm{~Hz}, 1 \mathrm{H}, \beta C \mathrm{H}), 6.99-7.35(\mathrm{~m}, 10 \mathrm{H}, \mathrm{ArH}), 7.49$ (s, $1 \mathrm{H}, \beta \mathrm{CH})$ ppm. ${ }^{13} \mathrm{C} \mathrm{NMR}\left(300 \mathrm{MHz}, \mathrm{CDCl}_{3}\right): \delta=12.91$ $\left(\mathrm{CH}_{3}\right), 13.62\left(\gamma \mathrm{CH}_{3}\right), 37.50\left(\beta_{\mathrm{CH}_{2}}\right), 43.89\left(\mathrm{CH}_{2}\right), 52.28$ $\left(\mathrm{OCH}_{3}\right), 53.07(\alpha \mathrm{CH}), 67.37\left(\mathrm{CH}_{2} \mathrm{Z}\right), 127.15(\mathrm{CH}), 127.80$ $(\mathrm{CH}), 128.04(\mathrm{CH}), 128.41(\mathrm{CH}), 128.59(\mathrm{CH}), 129.04$ $(\mathrm{CH}), 133.45(\mathrm{C}), 134.62(\mathrm{CH}), 135.58(\mathrm{C}), 136.23(\mathrm{C})$, $155.02(\mathrm{C}=\mathrm{O}), 163.79(\mathrm{C}=\mathrm{O}), 171.61(\mathrm{C}=\mathrm{O}) \mathrm{ppm} . \mathrm{m} / \mathrm{z}$ (HRESI-MS) 425.20710, $\left([\mathrm{M}+\mathrm{H}]^{+}, \mathrm{C}_{24} \mathrm{H}_{28} \mathrm{~N}_{2} \mathrm{O}_{5}\right.$, requires 425.20765).
Synthesis of Z-N(Et)-Z-APhe-L-Phe-OMe (19b) The general procedure described above was followed using Z-N(Et)-Z$\Delta$ Phe-OH $(Z-17 b)(0.321 \mathrm{~g}, 0.988 \mathrm{mmol})$ as reactant to afford 19b $(0.386 \mathrm{~g}, 80 \%)$ as a light yellow oil that failed to crystallize. ${ }^{1} \mathrm{H}$ NMR $\left(400 \mathrm{MHz}, \mathrm{CDCl}_{3}\right): \delta=0.95$ (br. s, $3 \mathrm{H}$, $\mathrm{CH}_{2} \mathrm{CH}_{3}$ ), 2.95-3.21 (m, $\left.4 \mathrm{H}, \mathrm{CH}_{2} \mathrm{CH}_{3}+\beta_{\mathrm{CH}_{2}}\right), 3.71$ (s, 3H, $\left.\mathrm{CH}_{3} \mathrm{OMe}\right), 4.82-5.30\left(\mathrm{~m}, 3 \mathrm{H}, \mathrm{CH}_{2} \mathrm{Z}+\alpha \mathrm{CH}\right), 6.46(\mathrm{~d}, J=$ $7.2 \mathrm{~Hz}, 1 \mathrm{H}, \mathrm{NH}), 7.01-7.32(\mathrm{~m}, 15 \mathrm{H}, \mathrm{ArH}), 7.49(\mathrm{~s}, 1 \mathrm{H}$, $\beta \mathrm{CH}) \mathrm{ppm} .{ }^{13} \mathrm{C} \mathrm{NMR}\left(400 \mathrm{MHz}, \mathrm{CDCl}_{3}\right): \delta=13.07\left(\mathrm{CH}_{3}\right)$, $37.52\left(\mathrm{CCH}_{2}\right), 43.68\left(\mathrm{CH}_{2}\right), 52.29\left(\mathrm{OCH}_{3}\right), 53.48(\alpha \mathrm{CH})$, $67.62\left(\mathrm{CH}_{2} \mathrm{Z}\right), 127.22(\mathrm{CH}), 127.90(\mathrm{CH}), 128.02(\mathrm{CH})$, $128.35(\mathrm{CH}), 128.69(\mathrm{C}), 128.78(\mathrm{CH}), 129.09(\mathrm{CH}), 129.43$ $(\mathrm{CH}), 129.53(\mathrm{CH}), 129.64(\mathrm{CH}), 131.25(\mathrm{C}), 132.91(\mathrm{CH})$, $135.51(\mathrm{C}), 136.04(\mathrm{C}), 155.16(\mathrm{C}=\mathrm{O}), 164.95(\mathrm{C}=\mathrm{O})$, $171.60(\mathrm{C}=\mathrm{O}) \mathrm{ppm} . \mathrm{m} / \mathrm{z}$ (HRESI-MS) 487.22309, $\left([\mathrm{M}+\mathrm{H}]^{+}\right.$, $\mathrm{C}_{29} \mathrm{H}_{30} \mathrm{~N}_{2} \mathrm{O}_{5}$, requires 487.22330).

\section{Results and Discussion}

An alternative to the use of strong electron withdrawing moieties to enhance the acidity of the $\mathrm{NH}$ proton and allow $\mathrm{N}$-ethylation of amino acid derivatives can be the use of a strong base. In the case of amino acids, a strong base will induce racemisation and thus limit the value of the products obtained. With dehydroamino acids this problem does not occur, so the strong base potassium tert-butoxide was tested as auxiliary base in $\mathrm{N}$-ethylation of dehydroamino acid derivatives with triethyloxonium tetrafluoroborate. Initially, the methyl ester of $N$-(tert-butyloxycarbonyl), $\beta$-bromo, dehydroaminobutyric acid, for which no ethylation was observed using the weak base $N, N$-diisopropylethylamine (Monteiro et al. 2011), was subject to treatment with 2.5 equiv. of triethyloxonium tetrafluoroborate using 3.5 equiv. of potassium tert-butoxide as base. In these conditions the reaction was complete in approximately 30 minutes allowing, the isolation of the $N$-ethyl dehydroamino acid derivative in $85 \%$ yield. Thus, all other substrates that previously had given no alkylation reaction or incomplete reaction using DIPEA (Monteiro et al. 2011), were subject to ethylation using potassium tert-butoxide as base. Thus, the methyl ester of $\beta, \beta$-dibromo dehydroalanine protected with Boc and the $Z$-isomers of the methyl esters of $\beta$-bromo, dehydroaminobutyric acid and dehydrophenylalanine protected with $\mathrm{Z}\left(\mathrm{NO}_{2}\right), \mathrm{Z}$ and Boc, were reacted with 2.5 equiv. of triethyloxonium tetrafluoroborate using 3.5 equiv. of potassium tert-butoxide as base (compounds $\mathbf{4 c}, \mathbf{5 a}-\mathbf{c}, \mathbf{6 a -}$ c, Scheme 1, Table 1).
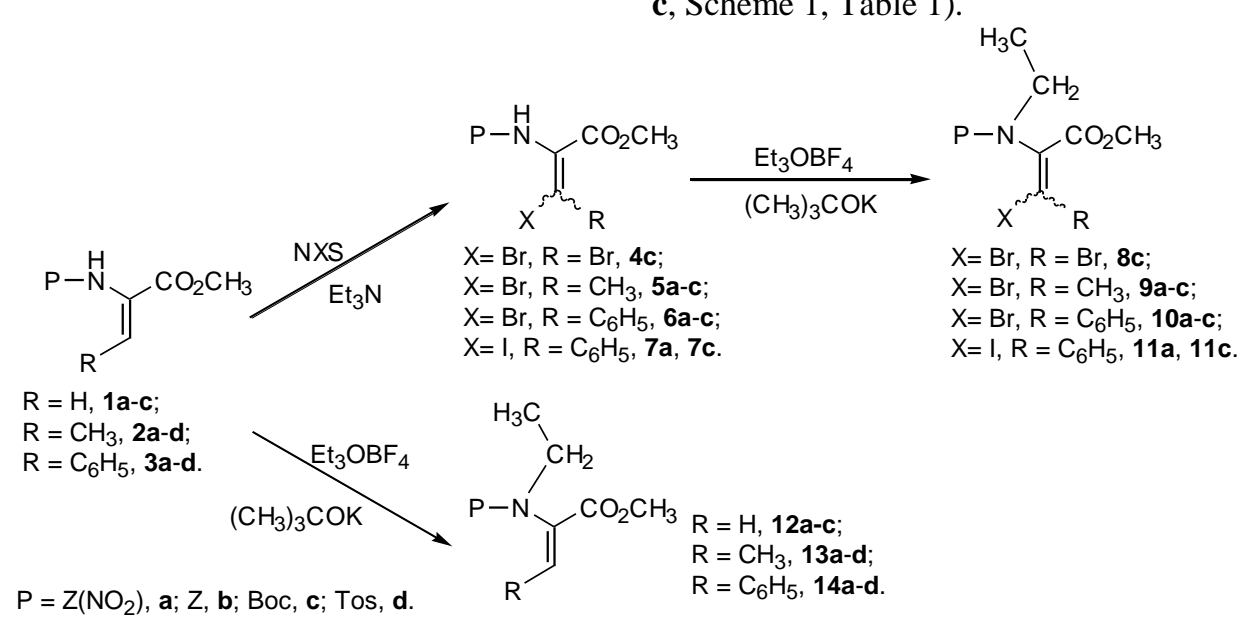

Scheme 1 Synthesis of $N$-ethyl dehydroamino acid derivatives 
For all the $\beta$-bromo dehydroamino acid derivatives, $N$ ethylation reactions were complete after approximately 30 minutes and the corresponding $N$-ethylated derivative could be isolated in high yield (Table 1). In two cases in which $E$-isomers of $\beta$-bromo dehydroamino acid derivatives were subject to $N$-alkylation (compounds $E$ 5a and $E$-5c) high yields in $N$-ethyl derivatives were obtained. The same procedure applied to two $\beta$-iodo derivatives of dehydrophenylalanine protected with the $\mathrm{Z}\left(\mathrm{NO}_{2}\right)$ and Boc groups (compounds Z-7a and Z-7c) gave the corresponding $N$-ethylated derivatives in excellent yields (Table 1).

Table 1 Results obtained in the $N$-ethylation of the methyl esters of $N$ protected $\beta, \beta$-dibromo and $\beta$-halo, $\beta$-substituted dehydroamino acids

\begin{tabular}{|c|c|c|}
\hline Reactant & Product & $\begin{array}{l}\text { Yield } \\
(\%)\end{array}$ \\
\hline Boc- $\Delta \mathrm{Ala}(\beta, \beta-\mathrm{Br})-\mathrm{OMe}, \mathbf{4 c}$ & Boc- $N(\mathrm{Et})-\Delta \mathrm{Ala}(\beta, \beta-\mathrm{Br})-\mathrm{OMe}, \mathbf{8 c}$ & 93 \\
\hline$-E-\Delta \mathrm{Abu}(\beta-\mathrm{Br})-\mathrm{OMe}, E-\mathbf{5 a}$ & $\mathrm{Z}\left(\mathrm{NO}_{2}\right)-N(\mathrm{Et})-E-\Delta \mathrm{Abu}(\beta-\mathrm{Br})-\mathrm{OMe} E$ & 94 \\
\hline $\mathrm{Z}\left(\mathrm{NO}_{2}\right)-\mathrm{Z}-\Delta \mathrm{Abu}(\beta-\mathrm{Br})-\mathrm{OMe}, \mathrm{Z}-\mathbf{5 a}$ & $\mathrm{Z}\left(\mathrm{NO}_{2}\right)-N(\mathrm{Et})-\mathrm{Z}-\Delta \mathrm{Abu}(\beta-\mathrm{Br})-\mathrm{OMe}, \mathrm{Z}-\mathbf{9 a}$ & 82 \\
\hline $\mathrm{Z}-\mathrm{Z}-\Delta \mathrm{Abu}(\beta-\mathrm{Br})-\mathrm{OMe}, \mathrm{Z}-\mathbf{5 b}$ & $\mathrm{Z}-N(\mathrm{Et})-Z-\Delta \mathrm{Abu}(\beta-\mathrm{Br})-\mathrm{OMe}, Z-\mathbf{9 b}$ & 86 \\
\hline Boc- $E$ & Boc- $N(\mathrm{Et}$ & 89 \\
\hline Boc- -2 & & 85 \\
\hline $\mathrm{Z}\left(\mathrm{NO}_{2}\right)-\mathrm{Z}-\Delta \mathrm{Phe}(\beta-\mathrm{Br})-\mathrm{C}$ & $\mathrm{Z}\left(\mathrm{NO}_{2}\right)-N(\mathrm{Et})-\mathrm{Z}-\Delta \mathrm{l}$ & 96 \\
\hline & & 95 \\
\hline Z-Z- $\Delta \mathrm{Phe}(\beta-\mathrm{Br})-\mathrm{OMe}, \mathrm{Z}-\mathbf{6 b}$ & $\mathrm{he}(\beta-\mathrm{Br})-\mathrm{OMe}, \mathrm{Z}-\mathbf{1 0 b}$ & 82 \\
\hline Boc-Z- $\Delta \mathrm{Phe}(\beta-\mathrm{Br})-\mathrm{OMe}, Z-\mathbf{6 c}$ & Boc- $N(\mathrm{Et})-Z-\Delta \mathrm{Phe}(\beta-\mathrm{Br})-\mathrm{OMe}, Z-\mathbf{1 0 c}$ & 88 \\
\hline Boc-Z- $\Delta$ Phe $(\beta-I)-O M e, Z-7 c$ & Boc- $N(\mathrm{Et})-Z-\Delta \mathrm{Phe}(\beta-\mathrm{I})-\mathrm{OMe}, Z-11 \mathrm{c}$ & 95 \\
\hline
\end{tabular}

$N$-Ethylation using the $\mathrm{Et}_{3} \mathrm{OBF}_{4} /$ DIPEA procedure of non-halogenated dehydroamino acids with amine protecting groups other than the 4-nitrobenzenesulfonyl group had been completely ineffective, even after several hours of reaction and addition of large excess of alkylating agent (Monteiro et al. 2011). However, the results obtained in ethylation of $\beta$-halo dehydroamino acid derivatives using as base potassium tert-butoxide, led us to reattempt the alkylation of non-halogenated dehydroamino acids with standard amine protecting groups. Thus, the methyl esters of dehydroalanine, dehydroaminobutyric acid and dehydrophenylalanine protected with $\mathrm{Z}\left(\mathrm{NO}_{2}\right), \mathrm{Z}$, Boc and 4-toluenesulfonyl (Tos) were subject to $N$-ethylation with triethyloxonium tetrafluoroborate using potassium tert-butoxide as base (compounds 1a-c, 2a-d, 3a-d, Scheme 1, Table 2). With all the dehydroamino acid derivatives the reactions were complete and the corresponding $N$-ethylated derivative could be isolated in high yield (Table 2).

Table 2 Results obtained in the $N$-ethylation of the methyl esters of $N$ protected dehydroamino acids

\begin{tabular}{|c|c|c|}
\hline Reactant & Product & $\begin{array}{c}\text { Yield } \\
(\%)\end{array}$ \\
\hline $\mathrm{Z}\left(\mathrm{NO}_{2}\right)-\Delta$ Ala-OMe, $\mathbf{1 a}$ & $\mathrm{Z}\left(\mathrm{NO}_{2}\right)-N(\mathrm{Et})-\Delta \mathrm{Ala}-\mathrm{OMe}, \mathbf{1 2 a}$ & 88 \\
\hline Z- $\Delta$ Ala-OMe, $\mathbf{1 b}$ & $\mathrm{Z}-N(\mathrm{Et})-\Delta \mathrm{Ala}-\mathrm{OMe}, \mathbf{1 2 b}$ & 91 \\
\hline Boc- $\Delta$ Ala-OMe, $1 c$ & Boc- $N(\mathrm{Et})-\Delta \mathrm{Ala}-\mathrm{OMe}, \mathbf{1 2 c}$ & 79 \\
\hline $\mathrm{Z}\left(\mathrm{NO}_{2}\right)-\mathrm{Z}-\Delta \mathrm{Abu}-\mathrm{OMe}, \mathrm{Z}-\mathbf{2 a}$ & $\mathrm{Z}\left(\mathrm{NO}_{2}\right)-N(\mathrm{Et})-\mathrm{Z}-\Delta \mathrm{Abu}-\mathrm{OMe}, \mathrm{Z}-\mathbf{1 3 a}$ & 90 \\
\hline Z-Z- $\Delta$ Abu-OMe, Z-2b & $\mathrm{Z}-N(\mathrm{Et})-Z-\Delta \mathrm{Abu}-\mathrm{OMe}, Z-\mathbf{1 3 b}$ & 93 \\
\hline Boc-Z- $\Delta$ Abu-OMe, Z- 2c & Boc- $N(\mathrm{Et})-Z-\Delta$ Abu-OMe, $Z-13 c$ & 92 \\
\hline Tos-Z- $\Delta$ Abu-OMe, Z-2d & Tos- $N(\mathrm{Et})-Z-\Delta \mathrm{Abu}-\mathrm{OMe}, Z-\mathbf{1 3 d}$ & 82 \\
\hline $\mathrm{Z}\left(\mathrm{NO}_{2}\right)-\mathrm{Z}-\Delta \mathrm{Phe}-\mathrm{OMe}, \mathrm{Z-3a}$ & $\mathrm{Z}\left(\mathrm{NO}_{2}\right)-N(\mathrm{Et})-Z-\Delta \mathrm{Phe}-\mathrm{OMe}, \mathrm{Z-14a}$ & 97 \\
\hline $\mathrm{Z}-\mathrm{Z}-\Delta \mathrm{Phe}-\mathrm{OMe}, \mathrm{Z}-\mathbf{3 b}$ & Z-N(Et)-Z- $\Delta$ Phe-OMe, Z-14b & 90 \\
\hline Boc-Z- $\Delta$ Phe-OMe, Z-3c & Boc- $N($ Et)-Z- $\Delta$ Phe-OMe, Z-14c & 92 \\
\hline Tos-Z- $\Delta$ Phe-OMe, Z-3d & Tos- $N($ Et)-Z- $\Delta$ Phe-OMe, Z-14d & 92 \\
\hline
\end{tabular}

By removal of the Nosyl protecting group from $\mathrm{N}$ Nosyl, $N$-ethyl amino acid methyl esters and reprotection of the amino function with the 9- fluorenylmethoxycarbonyl group (Fmoc), Liguori demonstrated the compatibility of this $N$-ethylation procedure with standard Fmoc chemistry (Belsito et al. 2001). However, further conversion of the methyl esters to acids is essential for other practical applications of these compounds, such as incorporation into peptides. Thus, representative methyl esters of $N$-ethyl dehydroamino acid derivatives were subject to cleavage of the methyl ester and subsequent coupling with an amino acid methyl ester. Three $N$-ethyl dehydroamino acid derivatives (compounds 12c, $Z$-13b and $Z-14 b$ ) were treated with a mixture of an aqueous solution of $\mathrm{NaOH}$ and dioxane, giving in all cases high yields in the corresponding $N$-protected, $N$-ethyl dehydroamino acid (compounds 15c, Z-16b and Z-17b, Scheme 2).

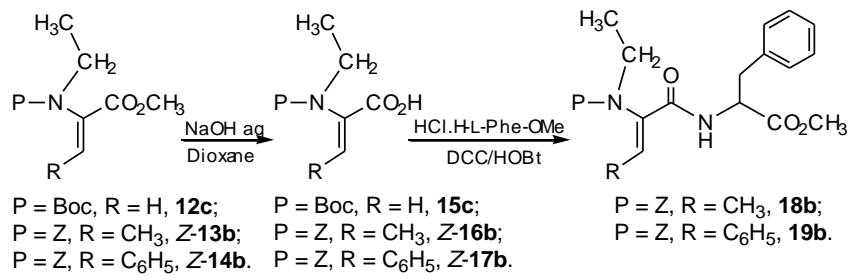

Scheme 2 Synthesis of $N$-ethyl dehydrodipeptide derivatives

Two of these dehydroamino acid derivatives (compounds Z-16b and Z-17b) were reacted with phenylalanine methyl ester using the standard DCC/HOBt procedure to give the corresponding $N$-ethyl dehydrodipeptide derivative in good yields (compounds $\mathbf{1 8 b}$ and 19b, Scheme 2).

\section{Conclusions}

A triethyloxonium tetrafluoroborate / potassium tertbutoxide alkylation procedure was applied for $\mathrm{N}$ ethylation of several methyl esters of $\beta, \beta$-dibromo dehydroalanine, $\beta$-bromo dehydroaminobutyric acid and $\beta$-halo dehydrophenylalanine, $\mathrm{N}$-protected with urethane groups. All $N$-ethyl, $\beta$-halo dehydroamino acid derivatives could be obtained in high yields. This method was also applied for $N$-ethylation of non-halogenated dehydroamino acids with urethane amine protecting groups and also the 4-toluenesulfonyl group. With all the dehydroamino acid derivatives the reactions were complete and the corresponding $N$-ethylated derivative could be isolated in high yields. Some of these $\mathrm{N}$ ethylated methyl ester derivatives were converted in high yields to their corresponding acids and coupled to an amino acid methyl ester to give $N$-ethyl dehydrodipeptide derivatives in good yields.

Thus, regardless of the nature of the dehydroamino acid and of the amine protecting group, this method constitutes a high yielding procedure for synthesis of $N$ ethylated dehydroamino acid derivatives, which can be further applied in peptide synthesis.

Acknowledgments Foundation for Science and Technology (FCT) - Portugal and Fundo Europeu de Desenvolvimento Regional (FEDER) for financial support to Chemistry Centre of University of Minho. The NMR spectrometer Bruker Avance $\mathrm{II}^{+} 400$ is part of the National NMR Network and was purchased in the framework of the National Program for 
Scientific Re-equipment; contract REDE/1517/RMN/2005, with funds from POCI 2010, FEDER and FCT.

\section{References}

Abreu AS, Ferreira PMT, Monteiro LS, Queiroz MJRP, Ferreira ICFR, Calhelha RC, Estevinho LM (2004) Synthesis of pure stereoisomers of benzo[b]thienyl dehydrophenylalanines by Suzuki cross-coupling. Preliminary studies of antimicrobial activity. Tetrahedron 60:11821-11828

Aurelio L, Brownlee RTC, Hughes AB (2004) Synthetic preparation of $N$-methyl- $\alpha$-amino acids. Chem. Rev. 104:5823-5846

Aydin M, Lucht N, Koenig WA, Lupp R, Jung G, Winkelmann $G$ (1985) Structure elucidation of the peptide antibiotics herbicolin A and B. Liebigs Ann. Chem. 1985:2285-2300

Belsito EL, De Marco R, Di Gioia ML, Liguori A, Perri F, Viscomi MC (2010) $N$-(4-Nitrophenylsulfonyl)- and $N$ (fluorenylmethoxycarbonyl)- $N$-ethyl amino acid methyl esters - a practical approach. Eur. J. Org. Chem. 2010:4245-4252

Bertj F, Ebert L, Gardossi L (1992) One-step stereospecific synthesis of $\alpha, \beta$-dehydroamino acids and dehydropeptides. Tetrahedron Lett. 33:8145-8148

Chatterjee C, Paul M, Xie L, Van Der Donk WA (2005) Biosynthesis and mode of action of lantibiotics. Chem. Rev. 105:633-684

Ferreira PMT, Maia HLS, Monteiro LS, Sacramento J (1999) High yielding synthesis of dehydroamino acid and dehydropeptide derivatives. J. Chem. Soc., Perkin Trans. 1 1999:3697-3703

Ferreira PMT, Maia HLS, Monteiro LS, Sacramento J (2001) Michael addition of thiols, carbon nucleophiles and amines to dehydroamino acid and dehydropeptide derivatives. J. Chem. Soc. Perkin Trans 1 2001:3167-3173

Ferreira PMT, Monteiro LS, Pereira G, Ribeiro L, Sacramento J, Silva L (2007) Reactivity of dehydroamino acids towards $N$-bromosuccinimide: synthesis of $\beta$-bromo and $\beta, \beta$ dibromodehydroamino acid derivatives and of 4imidazolidinones. Eur. J. Org. Chem. 2007: 5934-5949

Gilon C, Dechantsreiter MA, Burkhart F, Friedler A, Kessler H (2003) Synthesis of $N$-alkylated peptides. In: HoubenWeyl: Methods of organic chemistry. Synthesis of peptides and peptidomimetics; Eds.: M. Goodman, A. Felix, L. Moroder, C. Toniolo; Thieme Verlag, Stuttgart, New York, E22c:215-271

Goetz G, Nakao Y, Scheuer PJ (1997) Two acyclic kahalalides from the sacoglossan mollusk Elysia rufescens. J. Nat. Prod. 60:562-567

Hamann MT, Scheuer PJ (1993) Kahalalide F: a bioactive depsipeptide from the sacoglossan mollusk Elysia rufescens and the green alga Bryopsis sp. J. Am. Chem. Soc 115:5825-5826

Jung G (1991) Lantibiotics: ribosomally synthesized biologically active polypeptides containing sulfide bridges and $\alpha, \beta$-didehydroamino acids. Angew. Chem., Int. Ed. Engl. 30:1051-1068

Kotha S (2003) The building block approach to unusual $\alpha$ amino acid derivatives and peptides. Acc. Chem. Res. 36:342-351

Monteiro LS, Kołomańska J, Suárez AC (2010) Synthesis of novel non-proteinogenic amino acids: $N$-ethyl- $\alpha, \beta-$ dehydroamino acid methyl esters. Eur. J. Org. Chem. 2010:6731-6735

Monteiro LS, Andrade JJ, Suárez AC (2011) Synthesis of new $N$-ethyl dehydroamino acid derivatives: $N$-ethyl $\beta, \beta$ dibromo, $N$-ethyl $\beta$-bromo $\beta$-substituted, $N$-ethyl $\beta, \beta$ - disubstituted $\mathrm{N}$-protected dehydroamino acid methyl esters. Eur. J. Org. Chem. DOI: 10.1002/ejoc.201100907

Palmer DE, Pattaroni C, Nunami K, Chadha RK, Goodman M, Wakamiyia T, Fukase K, Horimoto S, Kitazawa M, Fujita H, Kubo A, Shiba T (1992) Effects of dehydroalanine on peptide conformations. J. Am. Chem. Soc. 114:5634-5642

Queiroz MJRP, Begouin A, Pereira G, Ferreira PMT (2008) New synthesis of methyl 5-aryl or heteroaryl pyrrole-2carboxylates by a tandem Sonogashira coupling/5-endodig-cyclization from $\beta$-iododehydroamino acid methyl esters and terminal alkynes. Tetrahedron, 64:10714-10720

Rilatt I, Caggiano L, Jackson RFW (2005) Development and applications of amino acid derived organometallics. Synlett, 2005:2701-2719

Silva NO, Abreu AS, Ferreira PMT, Monteiro LS, Queiroz MJRP (2002) Synthesis using Suzuki cross coupling of sulfur analogues of dehydrotryptophan with definite stereochemistry. Eur. J. Org. Chem. 2002:2524-2528

Silva NO, Abreu AS, Ferreira PMT, Queiroz MJRP (2003) Synthesis and intramolecular cyclization of novel $\beta, \beta$-bis(benzo $[b]$ thienyl)dehydroalanine derivatives. Tetrahedron Lett. 44:3377-3379

Wenger RM (1985) Synthesis of cyclosporine and analogues: structural requirements for immunosuppressive activity. Angew. Chem., Int. Ed. Engl. 24:77-85 